\title{
Search for a singly produced third-generation scalar leptoquark decaying to a $\tau$ lepton and a bottom quark in proton-proton collisions at $\sqrt{s}=13 \mathrm{TeV}$
}

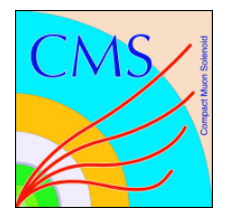

\section{The CMS collaboration}

E-mail: cms-publication-committee-chair@cern.ch

ABSTRACT: A search is presented for a singly produced third-generation scalar leptoquark decaying to a $\tau$ lepton and a bottom quark. Associated production of a leptoquark and a $\tau$ lepton is considered, leading to a final state with a bottom quark and two $\tau$ leptons. The search uses proton-proton collision data at a center-of-mass energy of $13 \mathrm{TeV}$ recorded with the CMS detector, corresponding to an integrated luminosity of $35.9 \mathrm{fb}^{-1}$. Upper limits are set at $95 \%$ confidence level on the production cross section of the third-generation scalar leptoquarks as a function of their mass. From a comparison of the results with the theoretical predictions, a third-generation scalar leptoquark decaying to a $\tau$ lepton and a bottom quark, assuming unit Yukawa coupling $(\lambda)$, is excluded for masses below $740 \mathrm{GeV}$. Limits are also set on $\lambda$ of the hypothesized leptoquark as a function of its mass. Above $\lambda=1.4$, this result provides the best upper limit on the mass of a third-generation scalar leptoquark decaying to a $\tau$ lepton and a bottom quark.

Keywords: Beyond Standard Model, Hadron-Hadron scattering (experiments)

ARXIV EPRINT: 1806.03472 


\section{Contents}

1 Introduction 1

2 The CMS detector 2

$\begin{array}{llr}3 & \text { Simulated samples } & 3\end{array}$

4 Event reconstruction $\quad 4$

5 Event selection $\quad 5$

$\begin{array}{llr}6 & \text { Background estimation } & 7\end{array}$

6.1 The $\ell \tau_{\mathrm{h}}$ channels $\quad 7$

6.2 The $\tau_{\mathrm{h}} \tau_{\mathrm{h}}$ channel 8

7 Systematic uncertainties and signal extraction $\quad 9$

7.1 Normalization uncertainties $\quad 9$

$\begin{array}{lll}7.2 & \text { Shape uncertainties } & 10\end{array}$

8 Results $\quad 10$

$\begin{array}{lll}9 & \text { Summary } & 13\end{array}$

$\begin{array}{ll}\text { The CMS collaboration } & 21\end{array}$

\section{Introduction}

Leptoquarks (LQs) are hypothetical color-triplet bosons, which carry both baryon and lepton quantum numbers and have fractional electric charge. They are predicted by many extensions of the standard model (SM) of particle physics, such as theories invoking grand unification [1-8], technicolor [9-11], or compositeness [12]. To satisfy experimental constraints on flavor changing neutral currents and other rare processes [13, 14], it is generally assumed that there would be three types of LQs, each type coupled to leptons and quarks of its same generation.

Third-generation scalar LQs have recently received considerable theoretical interest, as their existence can explain the anomaly in the $\overline{\mathrm{B}} \rightarrow \mathrm{D} \tau \bar{\nu}$ and $\overline{\mathrm{B}} \rightarrow \mathrm{D}^{*} \tau \bar{\nu}$ decay rates reported by the BaBar [15, 16], Belle [17-22], and LHCb [23] Collaborations. These decay rates collectively deviate from the SM predictions by about four standard deviations [24], and large couplings to third-generation quarks and leptons could explain this anomaly [25-28]. The LQ could also provide consistent explanations for other anomalies in B physics reported by LHCb [29-34] and Belle [35]. 

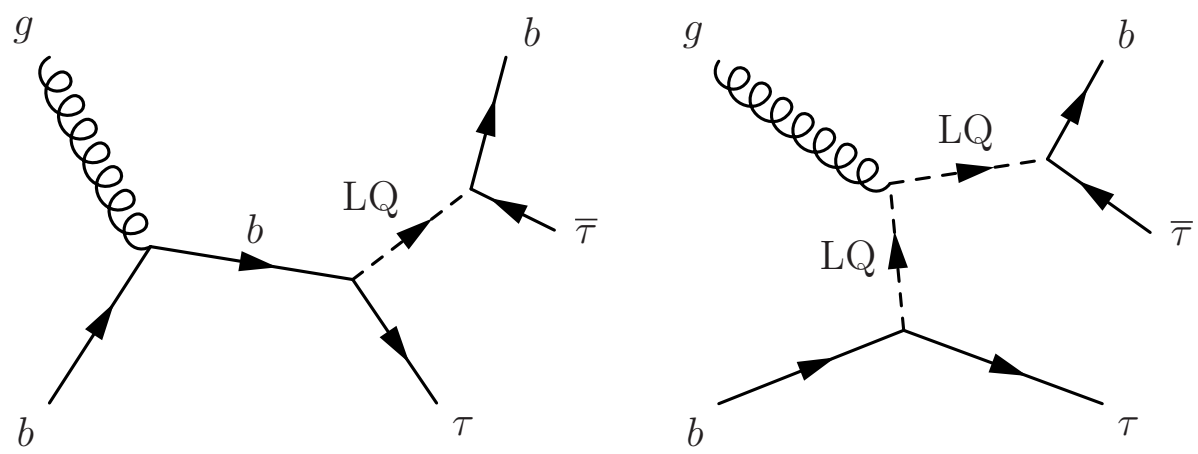

Figure 1. Leading order Feynman diagrams for the single production of third-generation LQs subsequently decaying to a $\tau$ lepton and a bottom quark, for the $s$-channel (left) and $t$-channel (right) processes.

The production cross sections and decay widths of LQs in proton-proton (pp) collisions are determined by the LQ's mass, $m_{\mathrm{LQ}}$; its branching fraction $\beta$ to a charged lepton and a quark; and the Yukawa coupling $\lambda$ of the LQ-lepton-quark vertex. Leptoquarks can be produced in pairs via gluon fusion or quark-antiquark annihilation, and singly via quark-gluon fusion. Pair production of LQs does not depend on $\lambda$, while single production does, and thus the sensitivity of searches for singly-produced LQs depends on $\lambda$. At lower masses, the cross section for pair production is greater than that for single production. However, the single-LQ production cross section decreases more slowly with increasing $m_{\mathrm{LQ}}$, eventually exceeding that for pair production. If the third-generation LQ is responsible for the observed B physics anomalies, then a large value of $\lambda$ is favored $\left(\lambda \sim m_{\mathrm{LQ}}\right.$ measured in $\mathrm{TeV}$ ), and the cross section for single production exceeds that for pair production for $m_{\mathrm{LQ}}$ greater than $1.0-1.5 \mathrm{TeV}[36]$.

The most stringent limits on the production of a third-generation LQ decaying to a $\tau$ lepton and a bottom quark comes from a search by the CMS Collaboration, in which a scalar LQ with mass below $850 \mathrm{GeV}$ was excluded in a search for LQ pair production in the $\ell \tau_{\mathrm{h}}$ bb final state [37]. Here, $\ell$ refers to a lepton (e or $\mu$ ) from $\tau$ lepton decay (35\% of the $\tau$ decays [38]), and $\tau_{\mathrm{h}}$ denotes a hadronically decaying $\tau$ lepton (65\% of the $\tau$ decays [38]). Another type of third-generation scalar LQ decaying to a $\tau$ lepton and a top quark is excluded for masses up to $900 \mathrm{GeV}$ [39].

This paper presents the first search that targets singly produced third-generation scalar LQs, each decaying to a $\tau$ lepton and a bottom quark. Feynman diagrams of the signal processes at leading order (LO) are shown in figure 1 . The final states $\ell \tau_{\mathrm{h}} \mathrm{b}$ and $\tau_{\mathrm{h}} \tau_{\mathrm{h}} \mathrm{b}$ are considered. The search is based on a data sample of pp collisions at a center-of-mass energy of $13 \mathrm{TeV}$ recorded by the CMS experiment at the CERN LHC in 2016, corresponding to an integrated luminosity of $35.9 \mathrm{fb}^{-1}$.

\section{The CMS detector}

The central feature of the CMS apparatus is a superconducting solenoid of $6 \mathrm{~m}$ internal diameter, providing a magnetic field of $3.8 \mathrm{~T}$. Within the solenoid volume, there are a silicon 
pixel and strip tracker, a lead tungstate crystal electromagnetic calorimeter (ECAL), and a brass and scintillator hadron calorimeter, each composed of a barrel and two endcap sections. Forward calorimeters extend the pseudorapidity coverage provided by the barrel and endcap detectors. Muons are measured in gas-ionization detectors embedded in the steel flux-return yoke outside the solenoid.

Events of interest are selected using a two-tiered trigger system [40]. The first level, composed of custom hardware processors, uses information from the calorimeters and muon detectors to select events at a rate of around $100 \mathrm{kHz}$ within a time interval of less than $4 \mu \mathrm{s}$. The second level, known as the high-level trigger, consists of a farm of processors running a version of the full event reconstruction software optimized for fast processing, and reduces the event rate to about $1 \mathrm{kHz}$ before data storage.

A more detailed description of the CMS detector, together with a definition of the coordinate system used and the relevant kinematic variables, can be found in ref. [41].

\section{Simulated samples}

Samples of simulated events are used to devise selection criteria and to estimate and validate background predictions. The LQ signals are generated at LO precision using version 2.6.0 of MAdGraph5_aMC@NLO [42] for $m_{\mathrm{LQ}}=200-1000$ (in steps of $100 \mathrm{GeV}$ ), 1200, and $1500 \mathrm{GeV}$. The particular LQ model used is $\tilde{R}_{2}$, as discussed in ref. [43]. The branching fraction is assumed to be $\beta=1$, i.e. the LQ always decays to a $\tau$ lepton and a bottom quark. The Yukawa coupling of the LQ to a $\tau$ lepton and a bottom quark is set to be $\lambda=1$. The width $\Gamma$ is calculated to be $\Gamma=m_{\mathrm{LQ}} \lambda^{2} /(16 \pi)$ [44], which is narrower than the experimental resolution over the considered search range. The signal samples are normalized to the cross section calculated at LO precision, multiplied by a $K$ factor to account for higher order contributions [45]. The $K$ factors are almost constant as a function of $m_{\mathrm{LQ}}$ and are approximately 1.4 for the bottom-quark-initiated diagrams considered in this analysis.

The main sources of background are the pair production of top quarks $(t \bar{t}), W$ and $\mathrm{Z}$ boson production in association with jets, denoted as "W+jets" and " $\mathrm{Z}+$ jets", diboson (WW, WZ, ZZ), single production of top quarks, and quantum chromodynamics (QCD) production of multijet events. The $\mathrm{W}+$ jets and $\mathrm{Z}+$ jets processes are simulated using the MADGRAPH5_aMC@NLO generator (v5_2.2.2 and v5_2.3.3) at LO precision with the MLM jet matching and merging scheme [46]. The same generator is also used for diboson production simulated at next-to-leading order (NLO) with the FxFx jet matching and

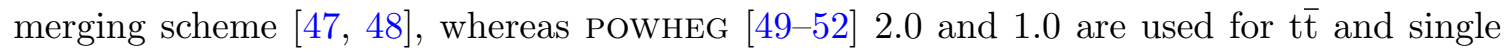
top quark production at NLO precision, respectively [53-55]. The t $\overline{\mathrm{t}}$ process is normalized using cross sections calculated at next-to-next-to-leading order in perturbative QCD [56].

The generators are interfaced with PYTHIA 8.212 [57] to model the parton showering and fragmentation, as well as the decay of the $\tau$ leptons. The PYTHIA parameters affecting the description of the underlying event are set to the CUETP8M1 tune [58]. The NNPDF3.0 parton distribution functions $[59,60]$ with the QCD order matching that of the matrix element calculations are used with all generators. 
Simulated events are processed with a model of the CMS detector based on GEANT4 [61] and are reconstructed with the same algorithms used for data. The effect of pileup, additional pp collisions within the same or adjacent bunch crossings, is taken into account by adding minimum bias events, generated with PYTHIA, to the hard scattering event. The additional events are weighted such that the frequency distribution matches that in data, with an average of approximately 23 interactions per bunch crossing [62].

\section{Event reconstruction}

The reconstruction of observed and simulated events uses a particle-flow (PF) algorithm [63], which combines the information from the CMS subdetectors to identify and reconstruct the particles emerging from pp collisions: charged and neutral hadrons, photons, muons, and electrons. Combinations of these PF objects are used to reconstruct higher-level objects such as jets, $\tau_{\mathrm{h}}$ candidates, or missing transverse momentum $\left(\vec{p}_{\mathrm{T}}^{\mathrm{miss}}\right)$, taken as the negative vector sum of the transverse momenta $\left(p_{\mathrm{T}}\right)$ of those jets.

The reconstructed vertex with the largest value of summed physics-object $p_{\mathrm{T}}^{2}$ is taken to be the primary pp collision vertex. In this case, the physics objects are the objects constructed by a jet finding algorithm $[64,65]$ applied to all charged tracks associated with the vertex, including tracks from lepton candidates, and the corresponding associated $\vec{p}_{\mathrm{T}}^{\text {miss }}$.

Electrons are identified with a multivariate (MVA) [66] discriminant combining several quantities describing the track quality, the shape of the energy deposits in the ECAL, and the compatibility of the measurements from the tracker and the ECAL [67]. The electrons must pass a cut-based discriminant to reject electrons coming from photon conversions. Muons are identified with requirements on the quality of the track reconstruction and on the number of measurements in the tracker and the muon systems [68]. Electron and muon candidates are required to have $p_{\mathrm{T}}>50 \mathrm{GeV}$. To reject leptons that do not come from the primary vertex and particles misidentified as leptons, a relative lepton isolation $I^{\ell}(\ell=\mathrm{e}, \mu)$ is defined as follows:

$$
I^{\ell} \equiv \frac{\sum_{\mathrm{ch}} p_{\mathrm{T}}+\max \left(0, \sum_{\text {neut }} p_{\mathrm{T}}-\frac{1}{2} \sum_{\mathrm{ch}, \mathrm{PU}} p_{\mathrm{T}}\right)}{p_{\mathrm{T}}^{\ell}} .
$$

In this expression, $\sum_{\mathrm{ch}} p_{\mathrm{T}}$ is the scalar sum of the $p_{\mathrm{T}}$ of the charged hadrons, electrons, and muons originating from the primary vertex and located in a cone of size $\Delta R=\sqrt{(\Delta \eta)^{2}+(\Delta \phi)^{2}}=0.03(0.04)$ centered on the electron (muon) direction, where $\eta$ is pseudorapidity and $\phi$ is azimuthal angle in radians. The sum $\sum_{\text {neut }} p_{\mathrm{T}}$ represents the same quantity for neutral hadrons and photons. The contribution of pileup photons and neutral hadrons is estimated from the scalar sum of the $p_{\mathrm{T}}$ of charged hadrons originating from pileup vertices, $\sum_{\mathrm{ch}, \mathrm{PU}} p_{\mathrm{T}}$. This sum is multiplied by a factor of $1 / 2$, which corresponds approximately to the ratio of neutral- to charged-hadron production in the hadronization process of inelastic pp collisions, as estimated from simulation. In this analysis, $I^{\mathrm{e}}<0.10$ $\left(I^{\mu}<0.15\right)$ is used as an isolation requirement for the electron (muon). With these cut-off values, the combined efficiency of identification and isolation is around 80 (95)\% for the electron (muon). Small differences, up to the $5 \%$ level, between data and simulation are corrected for by applying scale factors to simulated events. 
Jets are reconstructed from $\mathrm{PF}$ candidates using an anti- $k_{\mathrm{T}}$ clustering algorithm with a distance parameter of 0.4, implemented in the FASTJET library [65, 69]. Charged PF candidates not associated with the primary vertex of the interaction are not considered when reconstructing jets. An offset correction is applied to jet energies to take into account the contribution from additional pp collisions within the same or nearby bunch crossings. Jet energy corrections are derived from simulation to bring the average measured response of jets to that of particle-level jets [70]. Further identification requirements are applied to distinguish genuine jets from those coming from pileup [71, 72]. In this analysis, jets are required to have $p_{\mathrm{T}}$ greater than $30 \mathrm{GeV}$ and $|\eta|$ less than 4.7 , and must be separated from the selected leptons by a $\Delta R$ of at least 0.5 . Jets originating from the hadronization of bottom quarks are identified (b tagged) using the combined secondary vertex algorithm [73], which exploits observables related to the long lifetime and large mass of B hadrons. The chosen $\mathrm{b}$ tagging working point corresponds to an identification efficiency of approximately $60 \%$ with a misidentification rate of approximately $1 \%$, for jets originating from light (up, down, charm, strange) quarks and gluons.

The $\tau_{\mathrm{h}}$ candidates are reconstructed with the hadron-plus-strips algorithm [74, 75], which is seeded with anti- $k_{\mathrm{T}}$ jets. This algorithm reconstructs $\tau_{\mathrm{h}}$ candidates in the oneprong, one-prong $+\pi^{0}(\mathrm{~s})$, and three-prong decay modes, based on the number of tracks and on the number of strips of ECAL crystals with energy deposits. An MVA-based discriminator, including isolation as well as lifetime information, is used to reduce the incidence of jets being misidentified as $\tau_{\mathrm{h}}$ candidates. The typical working point used in this analysis has an efficiency $\approx 60 \%$ for a genuine $\tau_{\mathrm{h}}$, with a misidentification rate for quark and gluon jets of $\approx 0.1 \%$.

Electrons and muons misidentified as $\tau_{\mathrm{h}}$ candidates are suppressed using criteria based on the consistency between the measurements in the tracker, the calorimeters, and the muon detectors. The criteria are optimized separately for each final state studied.

All particles reconstructed in the event are used in the determination of $\vec{p}_{\mathrm{T}}^{\text {miss }}$ [76]. The calculation takes into account jet energy corrections. Corrections are applied to correct for the mismodeling of $\vec{p}_{\mathrm{T}}^{\text {miss }}$ in the simulated samples of the $\mathrm{Z}+$ jets and $\mathrm{W}+$ jets processes. The corrections are performed on the variable defined as the vectorial difference between the measured $\vec{p}_{\mathrm{T}}^{\text {miss }}$ and the total $p_{\mathrm{T}}$ of neutrinos originating from the decay of the $\mathrm{W}$ or $\mathrm{Z}$ boson.

\section{Event selection}

The search for scalar LQs is performed in three channels, each containing a b-tagged jet. Channels containing in addition an electron or a muon, together with a $\tau_{\mathrm{h}}$ candidate are labeled $\mathrm{e} \tau_{\mathrm{h}}$ and $\mu \tau_{\mathrm{h}}$, and collectively referred to as $\ell \tau_{\mathrm{h}}$. The third channel, which has two $\tau_{\mathrm{h}}$ candidates in addition to the b-tagged jet, is labeled $\tau_{\mathrm{h}} \tau_{\mathrm{h}}$. The selection criteria have been optimized based on the expected sensitivity to a single-LQ signal.

Firstly, events are required to be compatible with $\tau \tau$ production. In the $\mathrm{e} \tau_{\mathrm{h}}\left(\mu \tau_{\mathrm{h}}\right)$ channel, events are selected using a trigger that requires an isolated electron (muon) with $p_{\mathrm{T}}>25(24) \mathrm{GeV}$. Offline, the selected electron (muon) is required to have $p_{\mathrm{T}}>50 \mathrm{GeV}$ and $|\eta|<2.1(2.4)$. The $\tau_{\mathrm{h}}$ candidate is required to have $p_{\mathrm{T}}>50 \mathrm{GeV}$ and $|\eta|<2.3$. In 


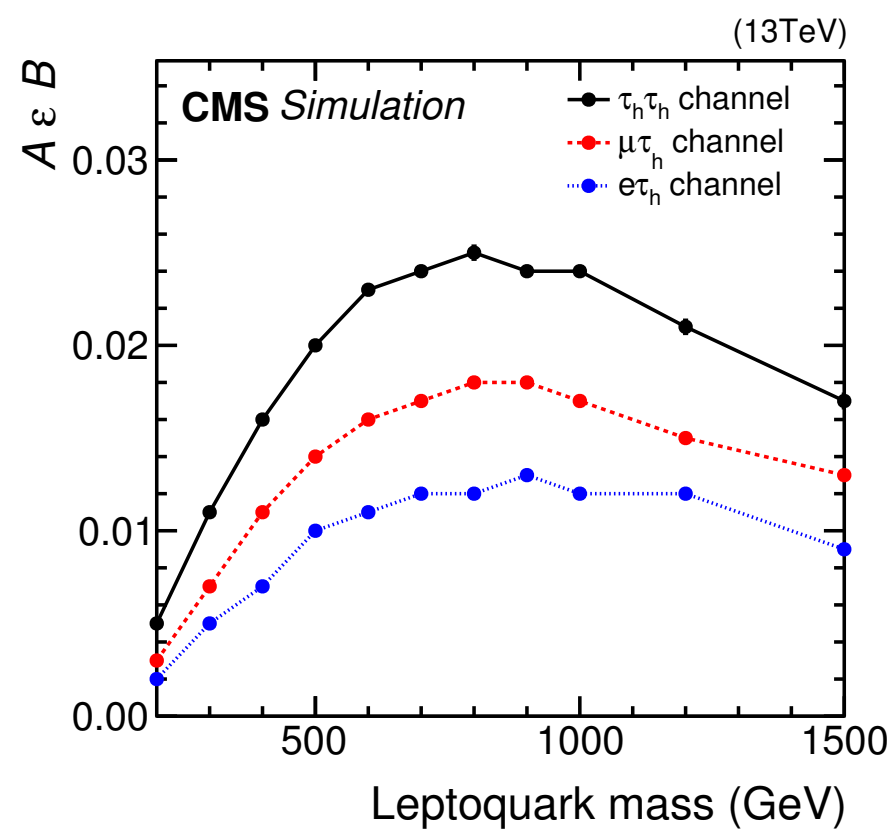

Figure 2. The product of acceptance, efficiency, and branching fraction as a function of $m_{\mathrm{LQ}}$ for the single production of LQs in each of the three channels considered: $\tau_{\mathrm{h}} \tau_{\mathrm{h}}$ (black solid line), $\mu \tau_{\mathrm{h}}$ (red dashed line), and $\mathrm{e} \tau_{\mathrm{h}}$ (blue dotted line).

the $\tau_{\mathrm{h}} \tau_{\mathrm{h}}$ channel, events are selected online by requiring two isolated $\tau_{\mathrm{h}}$ candidates with $p_{\mathrm{T}}>35 \mathrm{GeV}$. Offline, both $\tau_{\mathrm{h}}$ candidates are required to have $p_{\mathrm{T}}>50 \mathrm{GeV}$ and $|\eta|<2.1$. The selected $\ell$ and $\tau_{\mathrm{h}}$ candidate, or two $\tau_{\mathrm{h}}$ candidates, must meet isolation requirements as detailed in section 4, have opposite-sign (OS) electric charges and be separated by $\Delta R>0.5$. They must also meet the requirement that the distance of closest approach to the primary vertex satisfies $\left|d_{z}\right|<0.2 \mathrm{~cm}$ along the beam direction, and $\left|d_{x y}\right|<0.045 \mathrm{~cm}$ in the transverse plane. Events with additional isolated muons or electrons $\left(p_{\mathrm{T}}>10 \mathrm{GeV}\right.$ and $|\eta|<2.4$ or 2.5) that pass a looser identification requirement are discarded to reduce $\mathrm{Z}+$ jets and diboson backgrounds and to avoid correlations between channels.

Further event selection is applied to increase the signal purity. Since signal events contain at least one energetic bottom quark jet coming from the LQ decay, at least one b-tagged jet with $p_{\mathrm{T}}>50 \mathrm{GeV}$ and $|\eta|<2.4$ is required. To reduce the $\mathrm{Z}+$ jets background, the invariant mass, $m_{\mathrm{vis}}$, of the $\ell$ and $\tau_{\mathrm{h}}$ candidate (two $\tau_{\mathrm{h}}$ candidates), is required to be greater than $85(95) \mathrm{GeV}$ in the $\ell \tau_{\mathrm{h}}\left(\tau_{\mathrm{h}} \tau_{\mathrm{h}}\right)$ channels.

The product of acceptance $(\mathcal{A})$, efficiency $(\varepsilon)$, and the branching fraction $(\mathcal{B})$ of the $\tau \tau$ to a specific final state ranges from 0.2 to $1.3 \%$ in the $\mathrm{e} \tau_{\mathrm{h}}$ channel for $m_{\mathrm{LQ}}$ between 200 and $1500 \mathrm{GeV}$. The $\varepsilon$ increases with increasing $m_{\mathrm{LQ}}$ due to the harder $p_{\mathrm{T}}$ spectra of the final state particles. Beyond $1000 \mathrm{GeV}$, however, $\varepsilon$ starts to decrease, mainly because of the lower b tagging efficiency. Similarly, $\mathcal{A} \varepsilon \mathcal{B}$ in the $\mu \tau_{\mathrm{h}}\left(\tau_{\mathrm{h}} \tau_{\mathrm{h}}\right)$ channels range from 0.3 to $1.8 \%$ ( 0.5 to $2.5 \%$ ). Figure 2 shows $\mathcal{A} \varepsilon \mathcal{B}$ for the signal, in each final state considered in this analysis, as a function of $m_{\mathrm{LQ}}$. 
After applying the event selection, an excess of events over the SM backgrounds is searched for using the distribution of the scalar $p_{\mathrm{T}}$ sum of all required final-state particles, $S_{\mathrm{T}}$, which is defined as $p_{\mathrm{T}}(\ell)+p_{\mathrm{T}}\left(\tau_{\mathrm{h}}\right)+p_{\mathrm{T}}$ (leading jet) for the $\ell \tau_{\mathrm{h}}$ channels, and $p_{\mathrm{T}}$ (leading $\left.\tau_{\mathrm{h}}\right)+p_{\mathrm{T}}$ (subleading $\left.\tau_{\mathrm{h}}\right)+p_{\mathrm{T}}$ (leading jet) for the $\tau_{\mathrm{h}} \tau_{\mathrm{h}}$ channel, where leading and subleading refer to $p_{\mathrm{T}}$. Because of the $p_{\mathrm{T}}$ threshold requirements, $S_{\mathrm{T}}$ is always greater than $150 \mathrm{GeV}$.

\section{Background estimation}

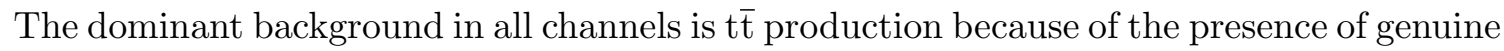
electrons, muons, $\tau$ leptons, and bottom quark jets produced in the t $\bar{t}$ decays. Additional backgrounds that satisfy the signal selection are $\mathrm{W}+$ jets, $\mathrm{Z}+$ jets, diboson, and single top quark processes, as well as QCD multijet events. In this section, background estimation methods and their validation are described separately for the $\ell \tau_{\mathrm{h}}$ and $\tau_{\mathrm{h}} \tau_{\mathrm{h}}$ channels.

\subsection{The $\ell \tau_{\mathrm{h}}$ channels}

The normalization and shape of the $t \bar{t}$ background are obtained from data, making use of an e $\mu$ control region (CR), containing events with an electron, a muon, and at least one b-tagged jet. The same $p_{\mathrm{T}}$ and $|\eta|$ requirements as in the signal region (SR) are placed on all three objects. The invariant mass of the selected electron and muon is required to be greater than $85 \mathrm{GeV}$. The purity of $\mathrm{t} \overline{\mathrm{t}}$ events in this $\mathrm{CR}$, estimated from simulation, is $92 \%$, with negligible signal contamination. A good agreement between data and simulation is found for both the normalization and the shape, validating the method used to estimate the $\mathrm{t} \overline{\mathrm{t}}$ background in the SR.

In order to allow for possible remaining mismodeling related to t $\bar{t}$ backgrounds, this $\mathrm{CR}$ is included in the maximum likelihood fit, as described in section 7, together with relevant nuisance parameters such as the $b$ tagging efficiency and $t \bar{t}$ cross section uncertainties. In this way, the normalization and the shape of the t $\overline{\mathrm{t}}$ backgrounds can be constrained from the data. This procedure also helps to constrain $t \bar{t}$ backgrounds in the $\tau_{\mathrm{h}} \tau_{\mathrm{h}}$ channel, although its contribution is less significant than in the $\ell \tau_{\mathrm{h}}$ channels.

For the $\mathrm{W}+$ jets background, the shape is taken from simulation, while the normalization is determined from data in a high $(>80 \mathrm{GeV})$ transverse mass $\left(m_{\mathrm{T}}\right)$ sideband; here $m_{\mathrm{T}}$ is defined as

$$
m_{\mathrm{T}}=\sqrt{2 p_{\mathrm{T}}^{\ell}\left|\vec{p}_{\mathrm{T}}^{\mathrm{miss}}\right|(1-\cos \Delta \phi)},
$$

where $p_{\mathrm{T}}^{\ell}$ is the lepton $p_{\mathrm{T}}$ and $\Delta \phi$ is the azimuthal angle between the lepton direction and $\vec{p}_{\mathrm{T}}^{\text {miss }}$. The normalization factor is calculated before the $\mathrm{b}$ tagging requirement is applied. A $30 \%$ uncertainty is assigned for the $\mathrm{W}+$ jets background estimate to account for the limited event counts in the high $m_{\mathrm{T}}$ sideband, as well as the extrapolation uncertainty to the SR.

The QCD multijet background, in which one of the jets is misidentified as the $\tau_{\mathrm{h}}$ candidate and another as the $\ell$, is small and is estimated using a CR where the $\ell$ and $\tau_{\mathrm{h}}$ candidate have same-sign (SS) electric charges. In this $\mathrm{CR}$, the QCD multijet yield is obtained by subtracting from the data the contribution of the $\mathrm{Z}+$ jets, $t \overline{\mathrm{t}}$, and $\mathrm{W}+$ jets 
processes. The expected contribution of the QCD multijet background in the OS SR is then derived by rescaling the yield obtained in the SS CR by a factor of 1.06 , which is measured using a pure QCD multijet sample obtained by inverting the lepton isolation requirement. To determine the uncertainty associated with this procedure, the measurement is repeated with several different $S_{\mathrm{T}}$ requirements. The maximum variation observed is $30 \%$, and this is taken to be the uncertainty in the QCD background estimate.

Minor backgrounds such as diboson and single top quark processes are estimated from the simulation.

\subsection{The $\tau_{\mathrm{h}} \tau_{\mathrm{h}}$ channel}

In the $\tau_{\mathrm{h}} \tau_{\mathrm{h}}$ channel, the shape and normalization of all background processes with genuine hadronic $\tau$ decays are estimated using simulated samples. The backgrounds concerned are $\mathrm{Z} \rightarrow \tau_{\mathrm{h}} \tau_{\mathrm{h}}$, and contributions from the $\mathrm{t} \overline{\mathrm{t}}$, diboson and single top quark processes.

Other backgrounds arising from jets misidentified as $\tau_{\mathrm{h}}$ candidates, most of which are from QCD multijet backgrounds, are estimated from CRs in data using the fake-factor method [77, 78]. An application region (AR) is defined containing the same selection criteria as in the $\mathrm{SR}$, except for an inverted $\tau_{\mathrm{h}}$ isolation requirement for one of the two $\tau_{\mathrm{h}}$ candidates. The AR is primarily populated by events with jets misidentified as $\tau_{\mathrm{h}}$ candidates, and has a contamination from genuine hadronic $\tau$ decays at the level of a few percent or below.

The ratio of the number of events with a misidentified $\tau_{\mathrm{h}}$ in the AR to the number in the SR (fake factor) is assumed to be the same as the ratio measured in samples with an $\mathrm{SS} \tau_{\mathrm{h}} \tau_{\mathrm{h}}$ pair. The fake factor is then applied to the number of events in the AR to estimate the number of events with a misidentified $\tau_{\mathrm{h}}$ in the SR. The fake factor is calculated as a function of the $p_{\mathrm{T}}$ and decay mode of the $\tau_{\mathrm{h}}$ candidate, and it ranges from 0.1 to 0.25 . In order to take combinatorial effects into account, a weight factor of 0.5 is applied. Since the presence of small backgrounds in the AR that contain a genuine $\tau_{\mathrm{h}}$ results in up to a $2 \%$ underestimation of the number of events with a misidentified $\tau_{\mathrm{h}}$ in the SR, a correction is applied based on the fractions of these processes in simulated events. Corresponding uncertainties are incorporated in the fit model, as described in section 7 .

The fake-factor method is tested in two validation regions (VRs); one is constructed by inverting the leading jet $p_{\mathrm{T}}$ requirement (i.e. $p_{\mathrm{T}}<50 \mathrm{GeV}$ ) and the other by using events with two $\tau_{\mathrm{h}}$ candidates that do not fulfill the tight isolation criteria used to define the SR. For both VRs, all other selection criteria are kept identical to the SR, except that the $\mathrm{b}$ tagging requirement is removed to increase the number of events. The signal contamination is negligible in both VRs. Agreement between data and simulation is found, within statistical uncertainties, demonstrating the validity of the fake-factor method.

Finally, small contributions coming from the $\mathrm{Z}+$ jets background are validated using the same event selection as in the SR, except that we require $m_{\mathrm{vis}}<95 \mathrm{GeV}$. Data and simulated events show agreement within statistical uncertainty. A $30 \%$ uncertainty is attributed to the $\mathrm{Z}+$ jets background yield due to the limited event count in this CR. 


\begin{tabular}{|c|c|c|c|}
\hline \multirow[t]{2}{*}{ Systematic source } & \multicolumn{3}{|c|}{ Uncertainty } \\
\hline & $\mathrm{e} \tau_{\mathrm{h}}$ & $\mu \tau_{\mathrm{h}}$ & $\tau_{\mathrm{h}} \tau_{\mathrm{h}}$ \\
\hline \multicolumn{4}{|l|}{ Normalization } \\
\hline Luminosity * & $2.5 \%$ & $2.5 \%$ & $2.5 \%$ \\
\hline Electron identification & $8 \%$ & - & - \\
\hline Electron trigger & $2 \%$ & - & - \\
\hline Muon identification & - & $2 \%$ & - \\
\hline Muon trigger & - & $2 \%$ & - \\
\hline$\tau_{\mathrm{h}}$ identification $*$ & $5 \%$ & $5 \%$ & $10 \%$ \\
\hline$\tau_{\mathrm{h}}$ trigger $*$ & - & - & $10 \%$ \\
\hline $\mathrm{b}$ tagging efficiency ${ }^{*}$ & $3 \%$ & $3 \%$ & $3 \%$ \\
\hline $\mathrm{b}$ tagging misidentification rate ${ }^{*}$ & $5 \%$ & $5 \%$ & $5 \%$ \\
\hline QCD multijet normalization & $30 \%$ & $30 \%$ & - \\
\hline $\mathrm{W}+$ jets normalization & $30 \%$ & $30 \%$ & - \\
\hline $\mathrm{Z} / \gamma^{*} \rightarrow \ell \ell$ cross section $*$ & $30 \%$ & $30 \%$ & $30 \%$ \\
\hline $\mathrm{t} \overline{\mathrm{t}} \operatorname{cross}$ section $*$ & $5.5 \%$ & $5.5 \%$ & $5.5 \%$ \\
\hline Diboson cross section $*$ & $6 \%$ & $6 \%$ & $6 \%$ \\
\hline Single top quark cross section $*$ & $5.5 \%$ & $5.5 \%$ & $5.5 \%$ \\
\hline $\mathrm{e} \rightarrow \tau_{\mathrm{h}}$ misidentification rate & $12 \%$ & - & - \\
\hline$\mu \rightarrow \tau_{\mathrm{h}}$ misidentification rate & - & $25 \%$ & - \\
\hline \multicolumn{4}{|l|}{ Shape } \\
\hline$\tau_{\mathrm{h}}$ energy scale ${ }^{*}$ & \multicolumn{3}{|c|}{ $\pm 3 \%$} \\
\hline$\tau_{\mathrm{h}}$ identification extrapolation $*$ & \multicolumn{3}{|c|}{$+5 \% p_{\mathrm{T}}\left(\tau_{\mathrm{h}}\right)$ and $-35 \% p_{\mathrm{T}}\left(\tau_{\mathrm{h}}\right)$} \\
\hline Jet energy scale $*$ & \multicolumn{3}{|c|}{ \pm 1 standard deviation $[70]$} \\
\hline Jet $\rightarrow \tau_{\mathrm{h}}$ misidentification rate ${ }^{*}$ & \multicolumn{3}{|c|}{ Described in the text (only $\ell \tau_{\mathrm{h}}$ channels) } \\
\hline Fake-factor method & \multicolumn{3}{|c|}{ Described in the text (only $\tau_{\mathrm{h}} \tau_{\mathrm{h}}$ channel) } \\
\hline Simulated sample size & \multicolumn{3}{|c|}{ Statistical uncertainty in individual bins } \\
\hline
\end{tabular}

Table 1. Summary of systematic uncertainties in the signal acceptance and background estimate. The uncertainties have been grouped into those affecting the normalization of distributions and those affecting the shape, and uncertainties marked with a * are treated as correlated among channels.

\section{Systematic uncertainties and signal extraction}

A binned maximum likelihood method is used for the signal extraction [79]. As discussed in section 5 , the $S_{\mathrm{T}}$ distribution for $S_{\mathrm{T}}$ greater than $150 \mathrm{GeV}$ is used as the final discriminant. The fit is performed simultaneously in the $\mathrm{e} \tau_{\mathrm{h}}, \mu \tau_{\mathrm{h}}$, and $\tau_{\mathrm{h}} \tau_{\mathrm{h}}$ signal regions, as well as in the $\mathrm{e} \mu$ control region, as defined in section 6 . Systematic uncertainties may affect the normalization and the shape of the $S_{\mathrm{T}}$ distribution of the signal and background processes. These uncertainties are represented by nuisance parameters in the fit, as described below. The relevant uncertainties are summarized in table 1.

\subsection{Normalization uncertainties}

The uncertainty in the integrated luminosity amounts to $2.5 \%$ [62] and affects the normalization of the signal and background processes that are based on simulation. Uncertainties 
in the electron identification and trigger efficiency amount to 8 and $2 \%$, respectively, while those in the muon identification and trigger efficiency amount to $2 \%$ each. The $\tau_{\mathrm{h}}$ identification and trigger efficiency have been measured using the "tag-and-probe" technique [74, 75] and an uncertainty of $5 \%$ per $\tau_{\mathrm{h}}$ candidate is assigned. The acceptance uncertainty due to the $\mathrm{b}$ tagging efficiency (misidentification rate) is taken to be 3 (5)\%. A $30 \%$ uncertainty is attributed to the $\mathrm{W}+$ jets, $\mathrm{Z}+$ jets, and QCD multijet backgrounds in the $\ell \tau_{\mathrm{h}}$ channels, as discussed in section 6 . The cross section uncertainties in the $t \bar{t}$, diboson, and single top quark processes are 5.5, 6.0, and 5.5\%, respectively. For events where electrons or muons are misidentified as $\tau_{\mathrm{h}}$ candidates, predominantly $\mathrm{Z} \rightarrow$ ee events in the $\mathrm{e} \tau_{\mathrm{h}}$ channel and $\mathrm{Z} \rightarrow \mu \mu$ events in the $\mu \tau_{\mathrm{h}}$ channel, rate uncertainties of 12 and $25 \%$, respectively, are allocated, based on the tag-and-probe method.

\subsection{Shape uncertainties}

The energy scales of the $\tau_{\mathrm{h}}$ candidate and the leading jet affect the shape of the $S_{\mathrm{T}}$ distribution, as well as the normalization of the signal and background processes. The uncertainty is estimated by varying the $\tau_{\mathrm{h}}$ and jet energies within their respective uncertainties and recomputing $S_{\mathrm{T}}$ after the final selection. The uncertainty in the $\tau_{\mathrm{h}}$ energy scale amounts to $3 \%$ [74], whereas the variations due to the jet energy scale are in the 1-2\% range, depending on the jet $p_{\mathrm{T}}$ and $\eta[70]$.

The uncertainty in the extrapolation of the $\tau_{\mathrm{h}}$ identification efficiency to higher $p_{\mathrm{T}} \mathrm{s}$ is treated as a shape uncertainty. It is proportional to $p_{\mathrm{T}}\left(\tau_{\mathrm{h}}\right)$ and has a value of $+5 \% /-35 \%$ at $p_{\mathrm{T}}\left(\tau_{\mathrm{h}}\right)=1 \mathrm{TeV}$. The effects of the uncertainties due to the electron and muon energy scales are found to be negligible. The probability of a jet being misidentified as a $\tau_{\mathrm{h}}$ candidate has been checked using a t $\bar{t}$ control region in data. The difference between data and simulated events is fit using a linear function, and its functional form, $1.2-0.004\left[\max \left(120, p_{\mathrm{T}}\right) \mathrm{GeV}\right]$, is considered as a one-sided shape uncertainty for the $\ell \tau_{\mathrm{h}}$ channels.

In the $\tau_{\mathrm{h}} \tau_{\mathrm{h}}$ channel, additional shape uncertainties related to the fake-factor method are considered. There are eight variations coming from factors such as the finite number of events in the samples, possible neglected effects in the fake factor determination, additional uncertainties in the correction of the SS to OS extrapolation, and the uncertainties in the background composition in the AR, which is estimated with simulated events. When added in quadrature, these additional uncertainties are of order $10 \%$.

Finally, uncertainties related to the finite number of simulated events, and to the limited number of events in data CRs, are taken into account. They are considered for all bins of the distributions that are used to extract the results. The binning of the histograms are adjusted such that the uncertainty, for a given bin, does not exceed $15 \%$. They are uncorrelated across the different samples, and across the bins of a single distribution.

\section{Results}

Figure 3 shows the $S_{\mathrm{T}}$ distributions after the combined fit to the $\mathrm{e} \tau_{\mathrm{h}}, \mu \tau_{\mathrm{h}}$, and $\tau_{\mathrm{h}} \tau_{\mathrm{h}}$ signal regions, as well as to the e $\mu$ control region. The background uncertainty bands on the histograms of simulated events represent the sum in quadrature of statistical and systematic 

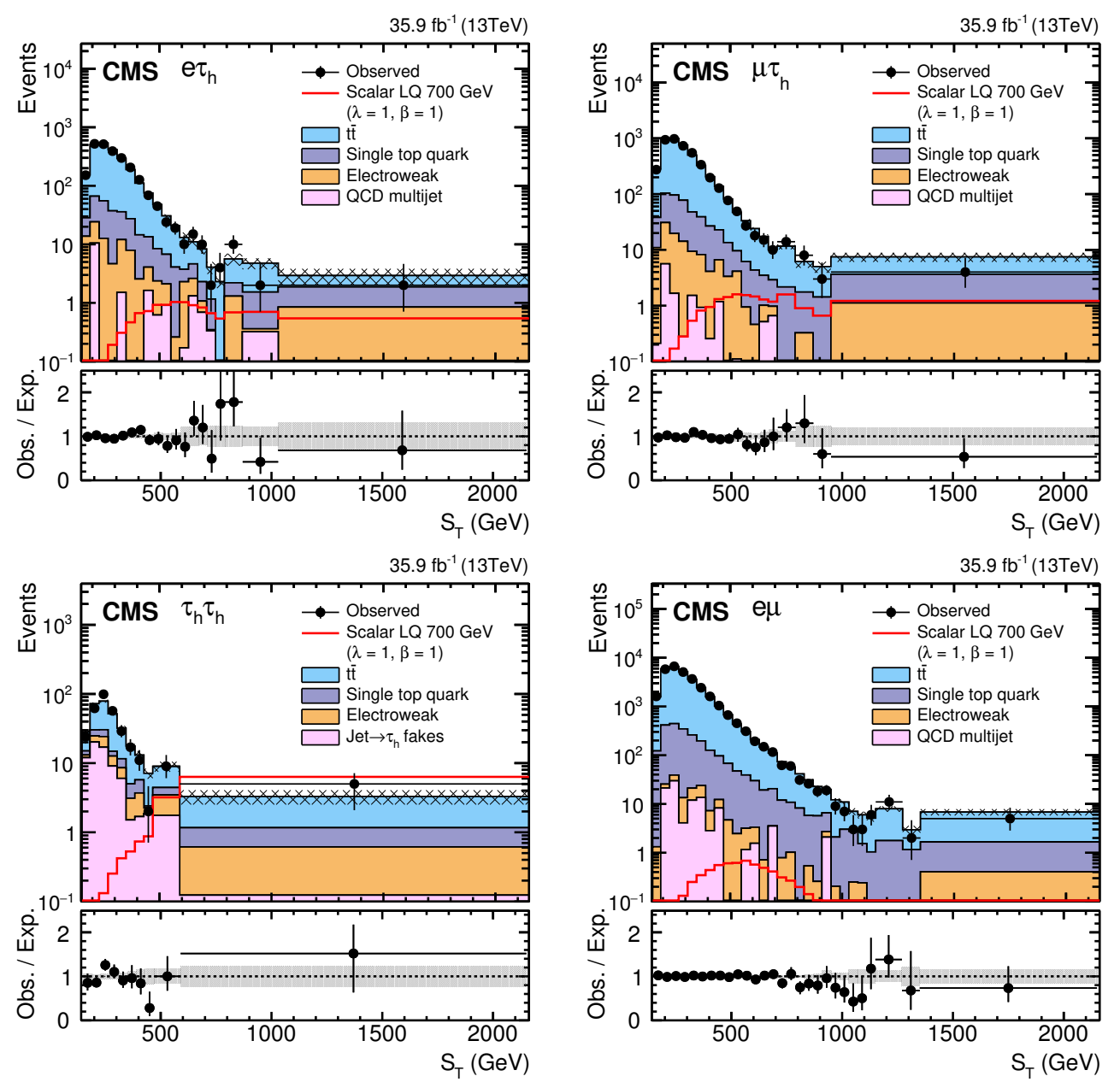

Figure 3. Observed $S_{\mathrm{T}}$ distribution in the e $\tau_{\mathrm{h}}$ (upper left), $\mu \tau_{\mathrm{h}}$ (upper right), and $\tau_{\mathrm{h}} \tau_{\mathrm{h}}$ (lower left) signal regions, as well as in the $\mathrm{e} \mu$ (lower right) control region, compared to the expected SM background contributions. The distribution labeled "electroweak" contains the contributions from $\mathrm{W}+$ jets, $\mathrm{Z}+$ jets, and diboson processes. The signal distributions for single-LQ production with mass $700 \mathrm{GeV}$ are overlaid to illustrate the sensitivity. For the signal normalization, $\lambda=1$ and $\beta=1$ are assumed. The background uncertainty bands represent the sum in quadrature of statistical and systematic uncertainties obtained from the fit. The lower panels show the ratio between the observed and expected events in each bin. In all plots, the horizontal and vertical error bars on the data points represent the bin widths and the Poisson uncertainties, respectively.

uncertainties, taking the full covariance matrix of all nuisance parameters into account. The dominant uncertainty in the background estimate comes from the limited event counts in simulated samples. However, this uncertainty is unimportant for $m_{\mathrm{LQ}}>500 \mathrm{GeV}$, where the mass limit is set, and the sensitivity is ultimately constrained by the size of the data sample.

Table 2 shows the event yields for a signal-enriched region with $S_{\mathrm{T}}>500 \mathrm{GeV}$, together with event yields expected for a representative LQ signal with $m_{\mathrm{LQ}}=700 \mathrm{GeV}$. 


\begin{tabular}{|lrrrc|}
\hline Process & \multicolumn{1}{c}{$\mathrm{e} \tau_{\mathrm{h}}$} & \multicolumn{1}{c|}{$\mu \tau_{\mathrm{h}}$} & \multicolumn{1}{c|}{$\tau_{\mathrm{h}} \tau_{\mathrm{h}}$} & \multicolumn{1}{c|}{$\mathrm{e} \mu$} \\
\hline $\mathrm{t} \overline{\mathrm{t}}$ & $114.8 \pm 2.9$ & $194.6 \pm 4.4$ & $6.7 \pm 1.0$ & $1895.2 \pm 14.4$ \\
Single top quark & $23.2 \pm 2.2$ & $36.6 \pm 2.6$ & $1.5 \pm 0.5$ & $263.4 \pm 6.8$ \\
Electroweak & $9.1 \pm 2.3$ & $10.9 \pm 3.1$ & $2.2 \pm 1.0$ & $16.0 \pm 2.4$ \\
QCD multijet & $4.5 \pm 4.6$ & $1.5 \pm 5.3$ & $1.9 \pm 0.6$ & $8.3 \pm 5.6$ \\
Total expected background & $151.6 \pm 6.3$ & $243.6 \pm 8.0$ & $12.3 \pm 1.7$ & $2182.9 \pm 17.0$ \\
LQ signal $\left(m_{\mathrm{LQ}}=700 \mathrm{GeV}, \lambda=1, \beta=1\right)$ & $8.8 \pm 0.3$ & $12.9 \pm 0.4$ & $9.5 \pm 1.2$ & $4.9 \pm 0.2$ \\
Observed data & 143 & 225 & 14 & 2147 \\
\hline
\end{tabular}

Table 2. Numbers of events observed in the $\mathrm{e} \tau_{\mathrm{h}}, \mu \tau_{\mathrm{h}}, \tau_{\mathrm{h}} \tau_{\mathrm{h}}$, and $\mathrm{e} \mu$ channels for $S_{\mathrm{T}}>500 \mathrm{GeV}$, compared to the background expectations and to the event yield expected for single-LQ processes with $m_{\mathrm{LQ}}=700 \mathrm{GeV}(\lambda=1$ and $\beta=1)$. The "electroweak" background contains the contributions from $\mathrm{W}+$ jets, $\mathrm{Z}+$ jets, and diboson processes. The uncertainties represent the sum in quadrature of statistical and systematic contributions, and are obtained using the binned maximum likelihood fit of the $S_{\mathrm{T}}$ distribution.

The data are consistent with the background-only (SM) hypothesis. In the $\tau_{\mathrm{h}} \tau_{\mathrm{h}}$ channel, one bin at around $250 \mathrm{GeV}$ shows a slight excess in data, corresponding to two standard deviations. This, however, has little impact on the results, as the sensitivity is dominated by the $S_{\mathrm{T}}$ tail, rather than the main part of the distribution.

We set an upper limit on the cross section times branching fraction $\beta$ as a function of $m_{\mathrm{LQ}}$, by using the asymptotic $\mathrm{CL}_{\mathrm{s}}$ modified frequentist criterion [79-82]. Figure 4 shows the observed and expected upper limits at $95 \%$ confidence level. The blue solid line corresponds to the theoretical cross sections [45], calculated with $\lambda=1$ and $\beta=1$. The intersection of the blue and the black lines determines the lower limit on $m_{\mathrm{LQ}}$. Assuming $\lambda=1$ and $\beta=1$, third-generation scalar LQs with masses below $740 \mathrm{GeV}$ are excluded at $95 \%$ confidence level, to be compared with an expected lower limit of $750 \mathrm{GeV}$.

The sensitivity of the analysis is dominated by the $\tau_{\mathrm{h}} \tau_{\mathrm{h}}$ channel, followed by the $\mu \tau_{\mathrm{h}}$ channel, and then the $\mathrm{e} \tau_{\mathrm{h}}$ channel. The better sensitivity in the $\tau_{\mathrm{h}} \tau_{\mathrm{h}}$ channel comes from the larger branching fraction of $\mathcal{B}\left(\tau \tau \rightarrow \tau_{\mathrm{h}} \tau_{\mathrm{h}}\right)=42 \%$, compared to $\mathcal{B}\left(\tau \tau \rightarrow \mu \tau_{\mathrm{h}}\right)=\mathcal{B}(\tau \tau \rightarrow$ $\left.\mathrm{e} \tau_{\mathrm{h}}\right)=21 \%$. Furthermore, the $\ell \tau_{\mathrm{h}}$ channels are contaminated by $\mathrm{t} \overline{\mathrm{t}} \rightarrow \mathrm{WWbb} \rightarrow \ell \tau_{\mathrm{h}} \nu \nu \mathrm{bb}$ process, in addition to the $\mathrm{t} \overline{\mathrm{t}} \rightarrow \mathrm{WWbb} \rightarrow \tau_{\ell} \tau_{\mathrm{h}} \nu \nu \mathrm{bb}$ background, which is not the case for the $\tau_{\mathrm{h}} \tau_{\mathrm{h}}$ channel. Here, $\tau_{\ell}$ denotes a leptonically-decaying $\tau$ lepton.

Since the single-LQ signal cross section scales with $\lambda^{2}$, it is straightforward to recast the results presented in figure 4 in terms of expected and observed upper limits on $\lambda$ as a function of $m_{\mathrm{LQ}}$, as shown in figure 5. Values of $\lambda$ up to 2.5 are considered, such that the width of the LQ signal stays narrow and to satisfy constraints from electroweak precision measurements [43]. Here we have made the assumption that the shape of the $S_{\mathrm{T}}$ distribution does not depend on $\lambda$ over the range of $\lambda$ used in the analysis. This assumption has been verified using simulated events. The blue band shows the preferred parameter space (95\% CL) for the scalar LQ preferred by the B physics anomalies: $\lambda=(0.95 \pm 0.50) m_{\mathrm{LQ}}(\mathrm{TeV})[36]$. The plot also shows the limit from the pair-produced LQ search overlaid as an orange vertical line, which does not depend on $\lambda$, as discussed in section 1 . For values of $\lambda>1.4$, the mass 


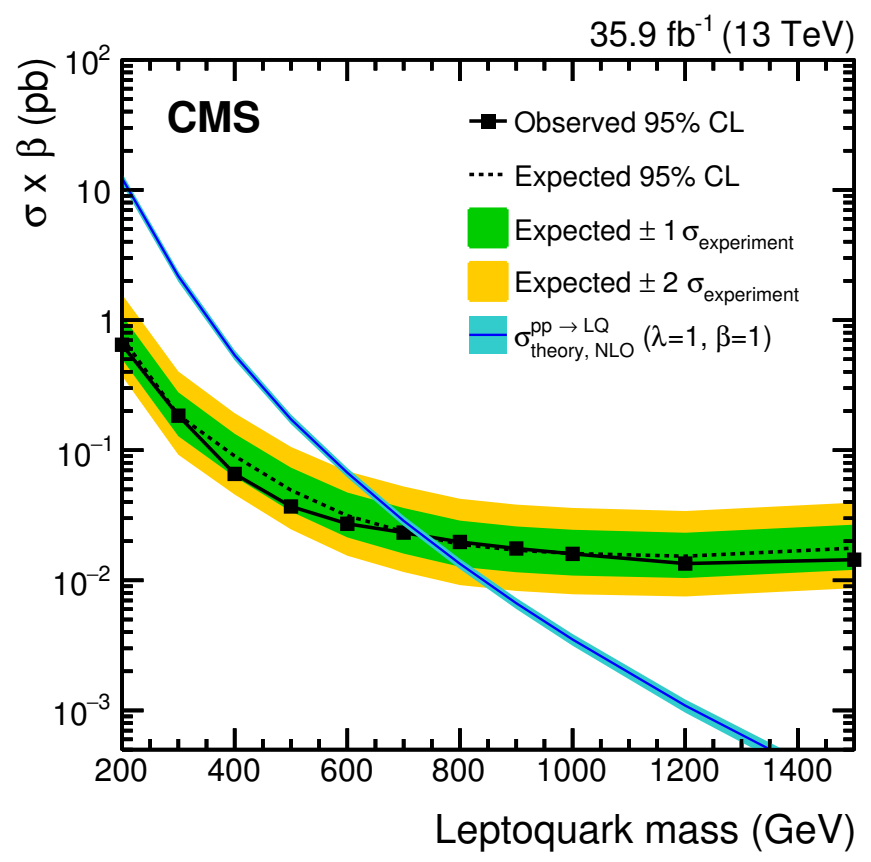

Figure 4. Observed (black solid) and expected (black dotted) limits at 95\% confidence level on the product of cross section $\sigma$ and branching fraction $\beta$, obtained from the combination of the $\mathrm{e} \tau_{\mathrm{h}}$, $\mu \tau_{\mathrm{h}}$, and $\tau_{\mathrm{h}} \tau_{\mathrm{h}}$ signal regions, as well as from the $\mathrm{e} \mu$ control region, as a function of the LQ mass. The green and yellow bands represent the one and two standard deviation uncertainties in the expected limits. The theory prediction is indicated by the blue solid line, together with systematic uncertainties due to the choice of PDF and renormalization and factorization scales [45], indicated by the blue band.

limit obtained by this analysis exceeds that of the search considering pair production [37] and provides the best upper limit on $m_{\mathrm{LQ}}$ of the third-generation scalar LQ decaying to a $\tau$ lepton and a bottom quark. For $\lambda=2.5$ and $\beta=1$, the observed and expected lower limits on mass are both $1050 \mathrm{GeV}$. This result, together with the pair-produced search, begins to constrain the region of parameter space implied by the B physics anomalies.

\section{Summary}

A search for singly produced third-generation scalar leptoquarks, each decaying to a $\tau$ lepton and a bottom quark has been presented. The final state of an electron or a muon plus one hadronically decaying $\tau$ lepton and the final state with two hadronically decaying $\tau$ leptons are explored. In all final states at least one energetic jet identified as originating from a bottom quark is required. The search is based on a data sample of proton-proton collisions at a center-of-mass energy of $13 \mathrm{TeV}$ recorded by the CMS detector, corresponding to an integrated luminosity of $35.9 \mathrm{fb}^{-1}$. The data are found to be in agreement with the standard model predictions. Upper limits as a function of the leptoquark mass are set on the third-generation scalar leptoquark production cross section. Results are compared with theoretical predictions to obtain lower limits on the leptoquark mass. Assuming the leptoquark always decays to a $\tau$ lepton and a bottom quark with unit Yukawa coupling 


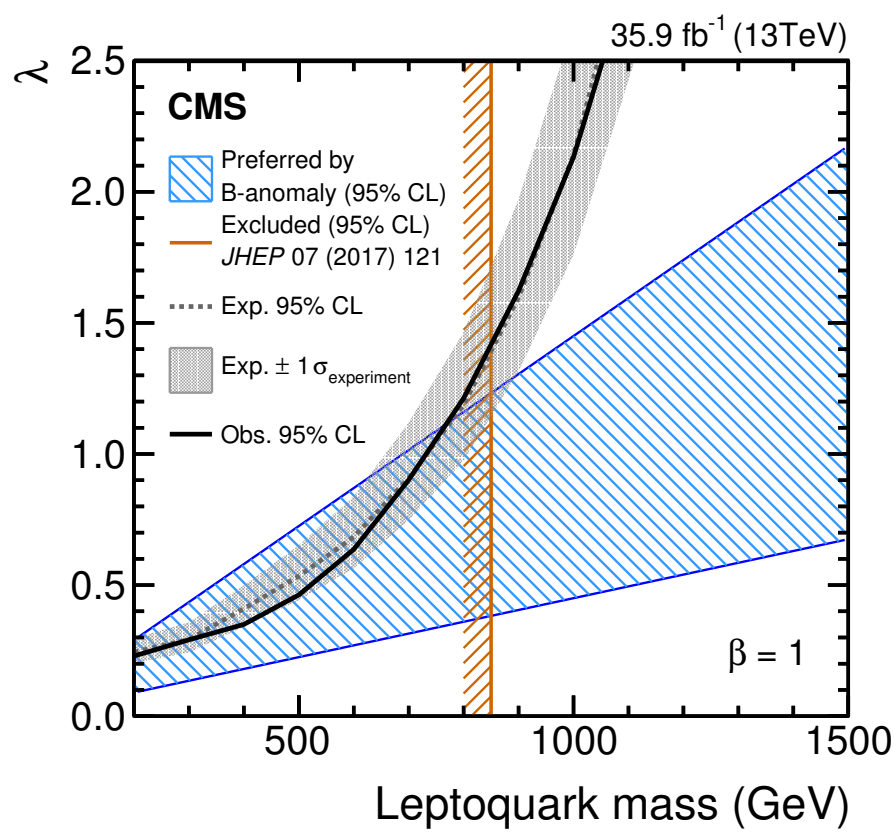

Figure 5. Expected and observed exclusion limits at 95\% confidence level on the Yukawa coupling $\lambda$ at the LQ-lepton-quark vertex, as a function of the LQ mass. A unit branching fraction $\beta$ of the LQ to a $\tau$ lepton and a bottom quark is assumed. The orange vertical line indicates the limit obtained from a search for pair-produced LQs decaying to $\ell \tau_{\mathrm{h}} \mathrm{bb}$ [37]. The left-hand side of the dotted (solid) line shows the expected (observed) exclusion region for the present analysis. The gray band shows \pm 1 standard deviations of the expected exclusion limit. The region with diagonal blue shading shows the parameter space preferred by one of the models proposed to explain anomalies observed in B physics [36].

$\lambda=1$, third-generation scalar leptoquarks with mass below $740 \mathrm{GeV}$ are excluded at $95 \%$ confidence level. Mass limits are also placed as a function of $\lambda$. For values of $\lambda>1.4$, the mass limit obtained by this analysis exceeds that of the search considering pair production and provides the best upper limit. For $\lambda=2.5$, leptoquarks are excluded in the mass range up to $1050 \mathrm{GeV}$. This is the first time that limits have been presented in the $\lambda$ versus mass plane, allowing the results to be considered in the preferred parameter space of models that invoke third-generation leptoquarks to explain anomalies observed in B hadron decays. These results thus demonstrate the important potential of single leptoquark production studies to complement pair production constraints on such models, as additional data become available.

\section{Acknowledgments}

We congratulate our colleagues in the CERN accelerator departments for the excellent performance of the LHC and thank the technical and administrative staffs at CERN and at other CMS institutes for their contributions to the success of the CMS effort. In addition, we gratefully acknowledge the computing centers and personnel of the Worldwide LHC Computing Grid for delivering so effectively the computing infrastructure essential to our 
analyses. Finally, we acknowledge the enduring support for the construction and operation of the LHC and the CMS detector provided by the following funding agencies: BMWFW and FWF (Austria); FNRS and FWO (Belgium); CNPq, CAPES, FAPERJ, FAPERGS, and FAPESP (Brazil); MES (Bulgaria); CERN; CAS, MoST, and NSFC (China); COLCIENCIAS (Colombia); MSES and CSF (Croatia); RPF (Cyprus); SENESCYT (Ecuador); MoER, ERC IUT, and ERDF (Estonia); Academy of Finland, MEC, and HIP (Finland); CEA and CNRS/IN2P3 (France); BMBF, DFG, and HGF (Germany); GSRT (Greece); NKFIA (Hungary); DAE and DST (India); IPM (Iran); SFI (Ireland); INFN (Italy); MSIP and NRF (Republic of Korea); LAS (Lithuania); MOE and UM (Malaysia); BUAP, CINVESTAV, CONACYT, LNS, SEP, and UASLP-FAI (Mexico); MBIE (New Zealand); PAEC (Pakistan); MSHE and NSC (Poland); FCT (Portugal); JINR (Dubna); MON, RosAtom, RAS, RFBR, and NRC KI (Russia); MESTD (Serbia); SEIDI, CPAN, PCTI, and FEDER (Spain); Swiss Funding Agencies (Switzerland); MST (Taipei); ThEPCenter, IPST, STAR, and NSTDA (Thailand); TUBITAK and TAEK (Turkey); NASU and SFFR (Ukraine); STFC (United Kingdom); DOE and NSF (U.S.A.). and SFFR (Ukraine); STFC (United Kingdom); DOE and NSF (U.S.A.).

Individuals have received support from the Marie-Curie program and the European Research Council and Horizon 2020 Grant, contract No. 675440 (European Union); the Leventis Foundation; the A. P. Sloan Foundation; the Alexander von Humboldt Foundation; the Belgian Federal Science Policy Office; the Fonds pour la Formation à la Recherche dans l'Industrie et dans l'Agriculture (FRIA-Belgium); the Agentschap voor Innovatie door Wetenschap en Technologie (IWT-Belgium); the F.R.S.-FNRS and FWO (Belgium) under the "Excellence of Science - EOS" - be.h project n. 30820817; the Ministry of Education, Youth and Sports (MEYS) of the Czech Republic; the Lendület ("Momentum") Program and the János Bolyai Research Scholarship of the Hungarian Academy of Sciences, the New National Excellence Program ÚNKP, the NKFIA research grants 123842, 123959, 124845, 124850 and 125105 (Hungary); the Council of Science and Industrial Research, India; the HOMING PLUS program of the Foundation for Polish Science, cofinanced from European Union, Regional Development Fund, the Mobility Plus program of the Ministry of Science and Higher Education, the National Science Center (Poland), contracts Harmonia 2014/14/M/ST2/00428, Opus 2014/13/B/ST2/02543, 2014/15/B/ST2/03998, and 2015/19/B/ST2/02861, Sonata-bis 2012/07/E/ST2/01406; the National Priorities Research Program by Qatar National Research Fund; the Programa Estatal de Fomento de la Investigación Científica y Técnica de Excelencia María de Maeztu, grant MDM-2015-0509 and the Programa Severo Ochoa del Principado de Asturias; the Thalis and Aristeia programs cofinanced by EU-ESF and the Greek NSRF; the Rachadapisek Sompot Fund for Postdoctoral Fellowship, Chulalongkorn University and the Chulalongkorn Academic into Its 2nd Century Project Advancement Project (Thailand); the Welch Foundation, contract C-1845; and the Weston Havens Foundation (U.S.A.).

Open Access. This article is distributed under the terms of the Creative Commons Attribution License (CC-BY 4.0), which permits any use, distribution and reproduction in any medium, provided the original author(s) and source are credited. 


\section{References}

[1] H. Georgi and S.L. Glashow, Unity of all elementary particle forces, Phys. Rev. Lett. 32 (1974) 438 [INSPIRE].

[2] J.C. Pati and A. Salam, Unified lepton-hadron symmetry and a gauge theory of the basic interactions, Phys. Rev. D 8 (1973) 1240 [INSPIRE].

[3] J.C. Pati and A. Salam, Lepton number as the fourth color, Phys. Rev. D 10 (1974) 275 [Erratum ibid. D 11 (1975) 703] [INSPIRE].

[4] H. Murayama and T. Yanagida, A viable SU(5) GUT with light leptoquark bosons, Mod. Phys. Lett. A 7 (1992) 147 [InSPIRE].

[5] H. Fritzsch and P. Minkowski, Unified interactions of leptons and hadrons, Annals Phys. 93 (1975) 193 [INSPIRE].

[6] G. Senjanović and A. Sokorac, Light leptoquarks in SO(10), Z. Phys. C 20 (1983) 255 [INSPIRE].

[7] P.H. Frampton and B.-H. Lee, SU(15) grand unification, Phys. Rev. Lett. 64 (1990) 619 [INSPIRE].

[8] P.H. Frampton and T.W. Kephart, Higgs sector and proton decay in SU(15) grand unification, Phys. Rev. D 42 (1990) 3892 [INSPIRE].

[9] S. Dimopoulos and L. Susskind, Mass without scalars, Nucl. Phys. B 155 (1979) 237 [INSPIRE].

[10] S. Dimopoulos, Technicolored signatures, Nucl. Phys. B 168 (1980) 69 [INSPIRE].

[11] E. Farhi and L. Susskind, Technicolor, Phys. Rept. 74 (1981) 277 [InSPIRE].

[12] B. Schrempp and F. Schrempp, Light leptoquarks, Phys. Lett. B 153 (1985) 101 [INSPIRE].

[13] W. Buchmüller and D. Wyler, Constraints on SU(5) type leptoquarks, Phys. Lett. B 177 (1986) 377 [INSPIRE].

[14] O.U. Shanker, $\pi \ell 2, K \ell 3$ and $K^{0}-\bar{K}^{0}$ constraints on leptoquarks and supersymmetric particles, Nucl. Phys. B 204 (1982) 375 [inSPIRE].

[15] BABAR collaboration, J.P. Lees et al., Evidence for an excess of $\bar{B} \rightarrow D^{(*)} \tau^{-} \bar{\nu}_{\tau}$ decays, Phys. Rev. Lett. 109 (2012) 101802 [arXiv:1205.5442] [INSPIRE].

[16] BABAR collaboration, J.P. Lees et al., Measurement of an excess of $\bar{B} \rightarrow D^{(*)} \tau^{-} \bar{\nu}_{\tau}$ decays and implications for charged Higgs bosons, Phys. Rev. D 88 (2013) 072012 [arXiv: 1303.0571] [INSPIRE].

[17] Belle collaboration, A. Matyja et al., Observation of $B^{0} \rightarrow D^{*-} \tau^{+} \nu_{\tau}$ decay at Belle, Phys. Rev. Lett. 99 (2007) 191807 [arXiv:0706.4429] [INSPIRE].

[18] Belle collaboration, A. Bozek et al., Observation of $B^{+} \rightarrow \bar{D}^{* 0} \tau^{+} \nu_{\tau}$ and evidence for $B^{+} \rightarrow \bar{D}^{0} \tau^{+} \nu_{\tau}$ at Belle, Phys. Rev. D 82 (2010) 072005 [arXiv:1005.2302] [InSPIRE].

[19] BELle collaboration, M. Huschle et al., Measurement of the branching ratio of $\bar{B} \rightarrow D^{(*)} \tau^{-} \bar{\nu}_{\tau}$ relative to $\bar{B} \rightarrow D^{(*)} \ell^{-} \bar{\nu}_{\ell}$ decays with hadronic tagging at Belle, Phys. Rev. D 92 (2015) 072014 [arXiv: 1507.03233] [INSPIRE].

[20] BeLle collaboration, Y. Sato et al., Measurement of the branching ratio of $\bar{B}^{0} \rightarrow D^{*+} \tau^{-} \bar{\nu}_{\tau}$ relative to $\bar{B}^{0} \rightarrow D^{*+} \ell^{-} \bar{\nu}_{\ell}$ decays with a semileptonic tagging method, Phys. Rev. D 94 (2016) 072007 [arXiv: 1607.07923] [INSPIRE]. 
[21] Belle collaboration, S. Hirose et al., Measurement of the $\tau$ lepton polarization and $R\left(D^{*}\right)$ in the decay $\bar{B} \rightarrow D^{*} \tau^{-} \bar{\nu}_{\tau}$, Phys. Rev. Lett. 118 (2017) 211801 [arXiv:1612.00529] [InSPIRE].

[22] Belle collaboration, S. Hirose et al., Measurement of the $\tau$ lepton polarization and $R\left(D^{*}\right)$ in the decay $\bar{B} \rightarrow D^{*} \tau^{-} \bar{\nu}_{\tau}$ with one-prong hadronic $\tau$ decays at Belle, Phys. Rev. D 97 (2018) 012004 [arXiv: 1709.00129] [INSPIRE].

[23] LHCb collaboration, Measurement of the ratio of branching fractions $\mathcal{B}\left(\bar{B}^{0} \rightarrow D^{*+} \tau^{-} \bar{\nu}_{\tau}\right) / \mathcal{B}\left(\bar{B}^{0} \rightarrow D^{*+} \mu^{-} \bar{\nu}_{\mu}\right)$, Phys. Rev. Lett. 115 (2015) 111803 [Erratum ibid. 115 (2015) 159901] [arXiv: 1506.08614] [INSPIRE].

[24] B. Dumont, K. Nishiwaki and R. Watanabe, LHC constraints and prospects for $S_{1}$ scalar leptoquark explaining the $\bar{B} \rightarrow D^{(*)} \tau \bar{\nu}$ anomaly, Phys. Rev. D 94 (2016) 034001 [arXiv: 1603.05248] [INSPIRE].

[25] M. Tanaka and R. Watanabe, New physics in the weak interaction of $\bar{B} \rightarrow D^{(*)} \tau \bar{\nu}$, Phys. Rev. D 87 (2013) 034028 [arXiv:1212.1878] [INSPIRE].

[26] Y. Sakaki, M. Tanaka, A. Tayduganov and R. Watanabe, Testing leptoquark models in $\bar{B} \rightarrow D^{(*)} \tau \bar{\nu}$, Phys. Rev. D 88 (2013) 094012 [arXiv:1309.0301] [InSPIRE].

[27] I. Doršner, S. Fajfer, N. Košnik and I. Nišandžić, Minimally flavored colored scalar in $\bar{B} \rightarrow D^{(*)} \tau \bar{\nu}$ and the mass matrices constraints, JHEP 11 (2013) 084 [arXiv:1306.6493] [INSPIRE].

[28] B. Gripaios, M. Nardecchia and S.A. Renner, Composite leptoquarks and anomalies in B-meson decays, JHEP 05 (2015) 006 [arXiv:1412.1791] [INSPIRE].

[29] LHCb collaboration, Differential branching fractions and isospin asymmetries of $B \rightarrow K^{(*)} \mu^{+} \mu^{-}$decays, JHEP 06 (2014) 133 [arXiv: 1403.8044] [INSPIRE].

[30] LHCb collaboration, Measurements of the $S$-wave fraction in $B^{0} \rightarrow K^{+} \pi^{-} \mu^{+} \mu^{-}$decays and the $B^{0} \rightarrow K^{*}(892)^{0} \mu^{+} \mu^{-}$differential branching fraction, JHEP 11 (2016) 047 [Erratum ibid. 04 (2017) 142] [arXiv: 1606.04731] [INSPIRE].

[31] LHCb collaboration, Angular analysis and differential branching fraction of the decay $B_{s}^{0} \rightarrow \phi \mu^{+} \mu^{-}$, JHEP 09 (2015) 179 [arXiv: 1506.08777] [InSPIRE].

[32] LHCb collaboration, Angular analysis of the $B^{0} \rightarrow K^{* 0} \mu^{+} \mu^{-}$decay using $3 \mathrm{fb}^{-1}$ of integrated luminosity, JHEP 02 (2016) 104 [arXiv: 1512.04442] [INSPIRE].

[33] LHCb collaboration, Test of lepton universality using $B^{+} \rightarrow K^{+} \ell^{+} \ell^{-}$decays, Phys. Rev. Lett. 113 (2014) 151601 [arXiv:1406.6482] [INSPIRE].

[34] LHCb collaboration, Test of lepton universality with $B^{0} \rightarrow K^{* 0} \ell^{+} \ell^{-}$decays, JHEP 08 (2017) 055 [arXiv: 1705.05802] [INSPIRE].

[35] Belle collaboration, S. Wehle et al., Lepton-Flavor-Dependent Angular Analysis of $B \rightarrow K^{*} \ell^{+} \ell^{-}$, Phys. Rev. Lett. 118 (2017) 111801 [arXiv:1612.05014] [INSPIRE].

[36] D. Buttazzo, A. Greljo, G. Isidori and D. Marzocca, B-physics anomalies: a guide to combined explanations, JHEP 11 (2017) 044 [arXiv: 1706.07808] [INSPIRE].

[37] CMS collaboration, Search for third-generation scalar leptoquarks and heavy right-handed neutrinos in final states with two tau leptons and two jets in proton-proton collisions at $\sqrt{s}=13 \mathrm{TeV}$, JHEP 07 (2017) 121 [arXiv:1703.03995] [INSPIRE].

[38] Particle Data Group collaboration, C. Patrignani et al., Review of Particle Physics, Chin. Phys. C 40 (2016) 100001 [InSPIRE]. 
[39] CMS collaboration, Search for third-generation scalar leptoquarks decaying to a top quark and a $\tau$ lepton at $\sqrt{s}=13 \mathrm{TeV}$, submitted to Eur. Phys. J. C [arXiv:1803.02864] [INSPIRE].

[40] CMS collaboration, The CMS trigger system, 2017 JINST 12 P01020 [arXiv:1609.02366] [INSPIRE].

[41] CMS collaboration, The CMS experiment at the CERN LHC, 2008 JINST 3 S08004 [INSPIRE].

[42] J. Alwall, R. Frederix, S. Frixione, V. Hirschi, F. Maltoni, O. Mattelaer et al., The automated computation of tree-level and next-to-leading order differential cross sections and their matching to parton shower simulations, JHEP 07 (2014) 079 [arXiv:1405.0301] [INSPIRE].

[43] I. Doršner, S. Fajfer, A. Greljo, J.F. Kamenik and N. Košnik, Physics of leptoquarks in precision experiments and at particle colliders, Phys. Rept. 641 (2016) 1 [arXiv:1603.04993] [INSPIRE].

[44] T. Plehn, H. Spiesberger, M. Spira and P.M. Zerwas, Formation and decay of scalar leptoquarks/squarks in e p collisions, Z. Phys. C 74 (1997) 611 [hep-ph/9703433] [INSPIRE].

[45] I. Doršner and A. Greljo, Leptoquark toolbox for precision collider studies, JHEP 05 (2018) 126 [arXiv: 1801.07641] [INSPIRE].

[46] J. Alwall et al., Comparative study of various algorithms for the merging of parton showers and matrix elements in hadronic collisions, Eur. Phys. J. C 53 (2008) 473 [arXiv:0706.2569] [INSPIRE].

[47] J.M. Campbell, R.K. Ellis and C. Williams, Vector boson pair production at the LHC, JHEP 07 (2011) 018 [arXiv: 1105.0020] [InSPIRE].

[48] R. Frederix and S. Frixione, Merging meets matching in MC@NLO, JHEP 12 (2012) 061 [arXiv: 1209.6215] [INSPIRE].

[49] P. Nason, A New method for combining NLO QCD with shower Monte Carlo algorithms, JHEP 11 (2004) 040 [hep-ph/0409146] [INSPIRE].

[50] S. Frixione, P. Nason and C. Oleari, Matching NLO QCD computations with Parton Shower simulations: the POWHEG method, JHEP 11 (2007) 070 [arXiv:0709.2092] [INSPIRE].

[51] S. Alioli, P. Nason, C. Oleari and E. Re, A general framework for implementing NLO calculations in shower Monte Carlo programs: the POWHEG BOX, JHEP 06 (2010) 043 [arXiv:1002.2581] [INSPIRE].

[52] S. Alioli, K. Hamilton, P. Nason, C. Oleari and E. Re, Jet pair production in POWHEG, JHEP 04 (2011) 081 [arXiv:1012.3380] [INSPIRE].

[53] S. Frixione, P. Nason and G. Ridolfi, A positive-weight next-to-leading-order Monte Carlo for heavy flavour hadroproduction, JHEP 09 (2007) 126 [arXiv:0707.3088] [INSPIRE].

[54] S. Alioli, P. Nason, C. Oleari and E. Re, NLO single-top production matched with shower in POWHEG: s- and t-channel contributions, JHEP 09 (2009) 111 [Erratum ibid. 02 (2010) 011] [arXiv:0907.4076] [INSPIRE].

[55] E. Re, Single-top Wt-channel production matched with parton showers using the POWHEG method, Eur. Phys. J. C 71 (2011) 1547 [arXiv: 1009.2450] [INSPIRE].

[56] M. Czakon and A. Mitov, Top++: A program for the calculation of the top-pair cross-section at Hadron colliders, Comput. Phys. Commun. 185 (2014) 2930 [arXiv:1112.5675] [INSPIRE]. 
[57] T. Sjöstrand et al., An introduction to PYTHIA 8.2, Comput. Phys. Commun. 191 (2015) 159 [arXiv: 1410.3012] [INSPIRE].

[58] CMS collaboration, Event generator tunes obtained from underlying event and multiparton scattering measurements, Eur. Phys. J. C 76 (2016) 155 [arXiv:1512.00815] [INSPIRE].

[59] NNPDF collaboration, R.D. Ball et al., Unbiased global determination of parton distributions and their uncertainties at NNLO and at LO, Nucl. Phys. B 855 (2012) 153 [arXiv:1107.2652] [INSPIRE].

[60] NNPDF collaboration, R.D. Ball et al., Parton distributions for the LHC Run II, JHEP 04 (2015) 040 [arXiv: 1410.8849] [INSPIRE].

[61] GEANT4 collaboration, S. Agostinelli et al., GEANT4 - a simulation toolkit, Nucl. Instrum. Meth. A 506 (2003) 250 [INSPIRE].

[62] CMS collaboration, CMS luminosity measurements for the 2016 data taking period, CMS-PAS-LUM-17-001 (2017).

[63] CMS collaboration, Particle-flow reconstruction and global event description with the CMS detector, 2017 JINST 12 P10003 [arXiv:1706.04965] [INSPIRE].

[64] M. Cacciari, G.P. Salam and G. Soyez, The anti-k $k_{t}$ jet clustering algorithm, JHEP 04 (2008) 063 [arXiv: 0802.1189] [INSPIRE].

[65] M. Cacciari, G.P. Salam and G. Soyez, FastJet User Manual, Eur. Phys. J. C 72 (2012) 1896 [arXiv: 1111.6097] [INSPIRE].

[66] A. Hocker et al., TMVA, the Toolkit for Multivariate Data Analysis with ROOT, PoS (ACAT) 040 [physics/0703039] [INSPIRE].

[67] CMS collaboration, Performance of electron reconstruction and selection with the CMS detector in proton-proton collisions at $\sqrt{s}=8 \mathrm{TeV}, 2015$ JINST $10 \mathrm{P} 06005$ [arXiv: 1502.02701] [INSPIRE].

[68] CMS collaboration, Performance of CMS muon reconstruction in pp collision events at $\sqrt{s}=7 \mathrm{TeV}, 2012$ JINST 7 P10002 [arXiv:1206.4071] [inSPIRE].

[69] M. Cacciari and G.P. Salam, Dispelling the $N^{3}$ myth for the $k_{t}$ jet-finder, Phys. Lett. B 641 (2006) 57 [hep-ph/0512210] [INSPIRE].

[70] CMS collaboration, Determination of jet energy calibration and transverse momentum resolution in CMS, 2011 JINST 6 P11002 [arXiv:1107.4277] [INSPIRE].

[71] CMS collaboration, Jet algorithms performance in 13 TeV data, CMS-PAS-JME-16-003 (2017).

[72] CMS collaboration, Pileup jet identification, CMS-PAS-JME-13-005 (2013).

[73] CMS collaboration, Identification of heavy-flavour jets with the CMS detector in pp collisions at $13 \mathrm{TeV}, 2018$ JINST 13 P05011 [arXiv: 1712.07158] [INSPIRE].

[74] CMS collaboration, Reconstruction and identification of $\tau$ lepton decays to hadrons and $\nu_{\tau}$ at CMS, 2016 JINST 11 P01019 [arXiv: 1510.07488] [INSPIRE].

[75] CMS collaboration, Performance of reconstruction and identification of tau leptons in their decays to hadrons and tau neutrino in LHC Run-2, CMS-PAS-TAU-16-002 (2016).

[76] CMS collaboration, MET performance in 8 TeV data, CMS-PAS-JME-12-002 (2013). 
[77] CMS collaboration, Search for additional neutral MSSM Higgs bosons in the $\tau \tau$ final state in proton-proton collisions at $\sqrt{s}=13 \mathrm{TeV}$, submitted to JHEP [arXiv: 1803.06553] [INSPIRE].

[78] CMS collaboration, Measurement of the $\mathrm{Z}^{*} \rightarrow \tau \tau$ cross section in pp collisions at $\sqrt{s}=13$ TeV and validation of $\tau$ lepton analysis techniques, submitted to Eur. Phys. J. C [arXiv: 1801.03535] [INSPIRE].

[79] ATLAS, CMS collaborations and the LHC Higgs Combination Group, Procedure for the LHC Higgs boson search combination in Summer 2011, CMS-NOTE-2011-005 (2011).

[80] G. Cowan, K. Cranmer, E. Gross and O. Vitells, Asymptotic formulae for likelihood-based tests of new physics, Eur. Phys. J. C 71 (2011) 1554 [Erratum ibid. C 73 (2013) 2501] [arXiv:1007.1727] [INSPIRE].

[81] T. Junk, Confidence level computation for combining searches with small statistics, Nucl. Instrum. Meth. A 434 (1999) 435 [hep-ex/9902006] [INSPIRE].

[82] A.L. Read, Presentation of search results: The CL $L_{s}$ technique, J. Phys. G 28 (2002) 2693 [INSPIRE]. 


\section{The CMS collaboration}

\section{Yerevan Physics Institute, Yerevan, Armenia}

A.M. Sirunyan, A. Tumasyan

\section{Institut für Hochenergiephysik, Wien, Austria}

W. Adam, F. Ambrogi, E. Asilar, T. Bergauer, J. Brandstetter, M. Dragicevic, J. Erö, A. Escalante Del Valle, M. Flechl, R. Frühwirth ${ }^{1}$, V.M. Ghete, J. Hrubec, M. Jeitler ${ }^{1}$, N. Krammer, I. Krätschmer, D. Liko, T. Madlener, I. Mikulec, N. Rad, H. Rohringer, J. Schieck ${ }^{1}$, R. Schöfbeck, M. Spanring, D. Spitzbart, A. Taurok, W. Waltenberger, J. Wittmann, C.-E. Wulz ${ }^{1}$, M. Zarucki

Institute for Nuclear Problems, Minsk, Belarus

V. Chekhovsky, V. Mossolov, J. Suarez Gonzalez

Universiteit Antwerpen, Antwerpen, Belgium

E.A. De Wolf, D. Di Croce, X. Janssen, J. Lauwers, M. Pieters, M. Van De Klundert, H. Van Haevermaet, P. Van Mechelen, N. Van Remortel

\section{Vrije Universiteit Brussel, Brussel, Belgium}

S. Abu Zeid, F. Blekman, J. D'Hondt, I. De Bruyn, J. De Clercq, K. Deroover, G. Flouris, D. Lontkovskyi, S. Lowette, I. Marchesini, S. Moortgat, L. Moreels, Q. Python, K. Skovpen, S. Tavernier, W. Van Doninck, P. Van Mulders, I. Van Parijs

Université Libre de Bruxelles, Bruxelles, Belgium

D. Beghin, B. Bilin, H. Brun, B. Clerbaux, G. De Lentdecker, H. Delannoy, B. Dorney, G. Fasanella, L. Favart, R. Goldouzian, A. Grebenyuk, A.K. Kalsi, T. Lenzi, J. Luetic, N. Postiau, E. Starling, L. Thomas, C. Vander Velde, P. Vanlaer, D. Vannerom, Q. Wang

\section{Ghent University, Ghent, Belgium}

T. Cornelis, D. Dobur, A. Fagot, M. Gul, I. Khvastunov², D. Poyraz, C. Roskas, D. Trocino, M. Tytgat, W. Verbeke, B. Vermassen, M. Vit, N. Zaganidis

\section{Université Catholique de Louvain, Louvain-la-Neuve, Belgium}

H. Bakhshiansohi, O. Bondu, S. Brochet, G. Bruno, C. Caputo, P. David, C. Delaere, M. Delcourt, B. Francois, A. Giammanco, G. Krintiras, V. Lemaitre, A. Magitteri, A. Mertens, M. Musich, K. Piotrzkowski, A. Saggio, M. Vidal Marono, S. Wertz, J. Zobec

\section{Centro Brasileiro de Pesquisas Fisicas, Rio de Janeiro, Brazil}

F.L. Alves, G.A. Alves, L. Brito, G. Correia Silva, C. Hensel, A. Moraes, M.E. Pol, P. Rebello Teles

\section{Universidade do Estado do Rio de Janeiro, Rio de Janeiro, Brazil}

E. Belchior Batista Das Chagas, W. Carvalho, J. Chinellato ${ }^{3}$, E. Coelho, E.M. Da Costa, G.G. Da Silveira ${ }^{4}$, D. De Jesus Damiao, C. De Oliveira Martins, S. Fonseca De Souza, H. Malbouisson, D. Matos Figueiredo, M. Melo De Almeida, C. Mora Herrera, L. Mundim, H. Nogima, W.L. Prado Da Silva, L.J. Sanchez Rosas, A. Santoro, A. Sznajder, M. Thiel, E.J. Tonelli Manganote ${ }^{3}$, F. Torres Da Silva De Araujo, A. Vilela Pereira 
Universidade Estadual Paulista ${ }^{a}$, Universidade Federal do ABC ${ }^{b}$, São Paulo, Brazil

S. Ahuja ${ }^{a}$, C.A. Bernardes ${ }^{a}$, L. Calligaris $^{a}$, T.R. Fernandez Perez Tomei ${ }^{a}$, E.M. Gregores $^{b}{ }$ P.G. Mercadante ${ }^{b}$, S.F. Novaes ${ }^{a}$, SandraS. Padula ${ }^{a}$, D. Romero Abad $^{b}$

Institute for Nuclear Research and Nuclear Energy, Bulgarian Academy of Sciences, Sofia, Bulgaria

A. Aleksandrov, R. Hadjiiska, P. Iaydjiev, A. Marinov, M. Misheva, M. Rodozov,

M. Shopova, G. Sultanov

University of Sofia, Sofia, Bulgaria

A. Dimitrov, L. Litov, B. Pavlov, P. Petkov

Beihang University, Beijing, China

W. Fang ${ }^{5}$, X. Gao ${ }^{5}$, L. Yuan

Institute of High Energy Physics, Beijing, China

M. Ahmad, J.G. Bian, G.M. Chen, H.S. Chen, M. Chen, Y. Chen, C.H. Jiang, D. Leggat, H. Liao, Z. Liu, F. Romeo, S.M. Shaheen ${ }^{6}$, A. Spiezia, J. Tao, C. Wang, Z. Wang, E. Yazgan,

H. Zhang, J. Zhao

State Key Laboratory of Nuclear Physics and Technology, Peking University, Beijing, China

Y. Ban, G. Chen, A. Levin, J. Li, L. Li, Q. Li, Y. Mao, S.J. Qian, D. Wang, Z. Xu

Tsinghua University, Beijing, China

Y. Wang

Universidad de Los Andes, Bogota, Colombia

C. Avila, A. Cabrera, C.A. Carrillo Montoya, L.F. Chaparro Sierra, C. Florez,

C.F. González Hernández, M.A. Segura Delgado

University of Split, Faculty of Electrical Engineering, Mechanical Engineering and Naval Architecture, Split, Croatia

B. Courbon, N. Godinovic, D. Lelas, I. Puljak, T. Sculac

University of Split, Faculty of Science, Split, Croatia

Z. Antunovic, M. Kovac

Institute Rudjer Boskovic, Zagreb, Croatia

V. Brigljevic, D. Ferencek, K. Kadija, B. Mesic, A. Starodumov ${ }^{7}$, T. Susa

University of Cyprus, Nicosia, Cyprus

M.W. Ather, A. Attikis, M. Kolosova, G. Mavromanolakis, J. Mousa, C. Nicolaou, F. Ptochos, P.A. Razis, H. Rykaczewski

Charles University, Prague, Czech Republic

M. Finger ${ }^{8}$, M. Finger Jr. ${ }^{8}$ 
Escuela Politecnica Nacional, Quito, Ecuador

E. Ayala

Universidad San Francisco de Quito, Quito, Ecuador

E. Carrera Jarrin

Academy of Scientific Research and Technology of the Arab Republic of Egypt, Egyptian Network of High Energy Physics, Cairo, Egypt

Y. Assran $^{9,10}$, S. Elgammal ${ }^{10}$, S. Khalil ${ }^{11}$

National Institute of Chemical Physics and Biophysics, Tallinn, Estonia

S. Bhowmik, A. Carvalho Antunes De Oliveira, R.K. Dewanjee, K. Ehataht, M. Kadastik, M. Raidal, C. Veelken

Department of Physics, University of Helsinki, Helsinki, Finland

P. Eerola, H. Kirschenmann, J. Pekkanen, M. Voutilainen

Helsinki Institute of Physics, Helsinki, Finland

J. Havukainen, J.K. Heikkilä, T. Järvinen, V. Karimäki, R. Kinnunen, T. Lampén, K. Lassila-Perini, S. Laurila, S. Lehti, T. Lindén, P. Luukka, T. Mäenpää, H. Siikonen, E. Tuominen, J. Tuominiemi

\section{Lappeenranta University of Technology, Lappeenranta, Finland}

T. Tuuva

IRFU, CEA, Université Paris-Saclay, Gif-sur-Yvette, France

M. Besancon, F. Couderc, M. Dejardin, D. Denegri, J.L. Faure, F. Ferri, S. Ganjour, A. Givernaud, P. Gras, G. Hamel de Monchenault, P. Jarry, C. Leloup, E. Locci, J. Malcles, G. Negro, J. Rander, A. Rosowsky, M.Ö. Sahin, M. Titov

Laboratoire Leprince-Ringuet, Ecole polytechnique, CNRS/IN2P3, Université Paris-Saclay, Palaiseau, France

A. Abdulsalam ${ }^{12}$, C. Amendola, I. Antropov, F. Beaudette, P. Busson, C. Charlot, R. Granier de Cassagnac, I. Kucher, S. Lisniak, A. Lobanov, J. Martin Blanco, M. Nguyen, C. Ochando, G. Ortona, P. Pigard, R. Salerno, J.B. Sauvan, Y. Sirois, A.G. Stahl Leiton, A. Zabi, A. Zghiche

Université de Strasbourg, CNRS, IPHC UMR 7178, Strasbourg, France J.-L. Agram ${ }^{13}$, J. Andrea, D. Bloch, J.-M. Brom, E.C. Chabert, V. Cherepanov, C. Collard, E. Conte ${ }^{13}$, J.-C. Fontaine ${ }^{13}$, D. Gelé, U. Goerlach, M. Jansová, A.-C. Le Bihan, N. Tonon, P. Van Hove

Centre de Calcul de l'Institut National de Physique Nucleaire et de Physique des Particules, CNRS/IN2P3, Villeurbanne, France

S. Gadrat 
Université de Lyon, Université Claude Bernard Lyon 1, CNRS-IN2P3, Institut de Physique Nucléaire de Lyon, Villeurbanne, France

S. Beauceron, C. Bernet, G. Boudoul, N. Chanon, R. Chierici, D. Contardo, P. Depasse, H. El Mamouni, J. Fay, L. Finco, S. Gascon, M. Gouzevitch, G. Grenier, B. Ille, F. Lagarde, I.B. Laktineh, H. Lattaud, M. Lethuillier, L. Mirabito, A.L. Pequegnot, S. Perries, A. Popov ${ }^{14}$, V. Sordini, M. Vander Donckt, S. Viret, S. Zhang

\section{Georgian Technical University, Tbilisi, Georgia}

A. Khvedelidze ${ }^{8}$

Tbilisi State University, Tbilisi, Georgia

D. Lomidze

RWTH Aachen University, I. Physikalisches Institut, Aachen, Germany

C. Autermann, L. Feld, M.K. Kiesel, K. Klein, M. Lipinski, M. Preuten, M.P. Rauch, C. Schomakers, J. Schulz, M. Teroerde, B. Wittmer, V. Zhukov ${ }^{14}$

RWTH Aachen University, III. Physikalisches Institut A, Aachen, Germany

A. Albert, D. Duchardt, M. Endres, M. Erdmann, T. Esch, R. Fischer, S. Ghosh, A. Güth,

T. Hebbeker, C. Heidemann, K. Hoepfner, H. Keller, S. Knutzen, L. Mastrolorenzo,

M. Merschmeyer, A. Meyer, P. Millet, S. Mukherjee, T. Pook, M. Radziej, H. Reithler,

M. Rieger, F. Scheuch, A. Schmidt, D. Teyssier

RWTH Aachen University, III. Physikalisches Institut B, Aachen, Germany

G. Flügge, O. Hlushchenko, B. Kargoll, T. Kress, A. Künsken, T. Müller, A. Nehrkorn, A. Nowack, C. Pistone, O. Pooth, H. Sert, A. Stahl ${ }^{15}$

Deutsches Elektronen-Synchrotron, Hamburg, Germany

M. Aldaya Martin, T. Arndt, C. Asawatangtrakuldee, I. Babounikau, K. Beernaert, O. Behnke, U. Behrens, A. Bermúdez Martínez, D. Bertsche, A.A. Bin Anuar, K. Borras ${ }^{16}$, V. Botta, A. Campbell, P. Connor, C. Contreras-Campana, F. Costanza, V. Danilov, A. De Wit, M.M. Defranchis, C. Diez Pardos, D. Domínguez Damiani, G. Eckerlin, T. Eichhorn, A. Elwood, E. Eren, E. Gallo ${ }^{17}$, A. Geiser, J.M. Grados Luyando, A. Grohsjean, P. Gunnellini, M. Guthoff, M. Haranko, A. Harb, J. Hauk, H. Jung, M. Kasemann, J. Keaveney, C. Kleinwort, J. Knolle, D. Krücker, W. Lange, A. Lelek, T. Lenz, K. Lipka, W. Lohmann ${ }^{18}$, R. Mankel, I.-A. Melzer-Pellmann, A.B. Meyer, M. Meyer, M. Missiroli, G. Mittag, J. Mnich, V. Myronenko, S.K. Pflitsch, D. Pitzl, A. Raspereza, M. Savitskyi, P. Saxena, P. Schütze, C. Schwanenberger, R. Shevchenko, A. Singh, N. Stefaniuk, H. Tholen, O. Turkot, A. Vagnerini, G.P. Van Onsem, R. Walsh, Y. Wen, K. Wichmann, C. Wissing, O. Zenaiev

\section{University of Hamburg, Hamburg, Germany}

R. Aggleton, S. Bein, L. Benato, A. Benecke, V. Blobel, M. Centis Vignali, T. Dreyer, E. Garutti, D. Gonzalez, J. Haller, A. Hinzmann, A. Karavdina, G. Kasieczka, R. Klanner, R. Kogler, N. Kovalchuk, S. Kurz, V. Kutzner, J. Lange, D. Marconi, J. Multhaup, M. Niedziela, D. Nowatschin, A. Perieanu, A. Reimers, O. Rieger, C. Scharf, P. Schleper, 
S. Schumann, J. Schwandt, J. Sonneveld, H. Stadie, G. Steinbrück, F.M. Stober, M. Stöver, D. Troendle, A. Vanhoefer, B. Vormwald

Karlsruher Institut fuer Technology

M. Akbiyik, C. Barth, M. Baselga, S. Baur, E. Butz, R. Caspart, T. Chwalek, F. Colombo, W. De Boer, A. Dierlamm, N. Faltermann, B. Freund, M. Giffels, M.A. Harrendorf, F. Hartmann ${ }^{15}$, S.M. Heindl, U. Husemann, F. Kassel ${ }^{15}$, I. Katkov ${ }^{14}$, S. Kudella, H. Mildner, S. Mitra, M.U. Mozer, Th. Müller, M. Plagge, G. Quast, K. Rabbertz, M. Schröder, I. Shvetsov, G. Sieber, H.J. Simonis, R. Ulrich, S. Wayand, M. Weber, T. Weiler, S. Williamson, C. Wöhrmann, R. Wolf

Institute of Nuclear and Particle Physics (INPP), NCSR Demokritos, Aghia Paraskevi, Greece

G. Anagnostou, G. Daskalakis, T. Geralis, A. Kyriakis, D. Loukas, G. Paspalaki, I. TopsisGiotis

National and Kapodistrian University of Athens, Athens, Greece

G. Karathanasis, S. Kesisoglou, P. Kontaxakis, A. Panagiotou, N. Saoulidou, E. Tziaferi, K. Vellidis

National Technical University of Athens, Athens, Greece

K. Kousouris, I. Papakrivopoulos, G. Tsipolitis

University of Ioánnina, Ioánnina, Greece

I. Evangelou, C. Foudas, P. Gianneios, P. Katsoulis, P. Kokkas, S. Mallios, N. Manthos, I. Papadopoulos, E. Paradas, J. Strologas, F.A. Triantis, D. Tsitsonis

MTA-ELTE Lendület CMS Particle and Nuclear Physics Group, Eötvös Loránd University, Budapest, Hungary

M. Bartók ${ }^{19}$, M. Csanad, N. Filipovic, P. Major, M.I. Nagy, G. Pasztor, O. Surányi, G.I. Veres

Wigner Research Centre for Physics, Budapest, Hungary

G. Bencze, C. Hajdu, D. Horvath ${ }^{20}$, Á. Hunyadi, F. Sikler, T.Á. Vámi, V. Veszpremi, G. Vesztergombi ${ }^{\dagger}$

Institute of Nuclear Research ATOMKI, Debrecen, Hungary

N. Beni, S. Czellar, J. Karancsi ${ }^{21}$, A. Makovec, J. Molnar, Z. Szillasi

Institute of Physics, University of Debrecen, Debrecen, Hungary

P. Raics, Z.L. Trocsanyi, B. Ujvari

Indian Institute of Science (IISc), Bangalore, India

S. Choudhury, J.R. Komaragiri, P.C. Tiwari

National Institute of Science Education and Research, HBNI, Bhubaneswar, India

S. Bahinipati22 , C. Kar, P. Mal, K. Mandal, A. $\operatorname{Nayak}^{23}$, D.K. Sahoo ${ }^{22}$, S.K. Swain 
Panjab University, Chandigarh, India

S. Bansal, S.B. Beri, V. Bhatnagar, S. Chauhan, R. Chawla, N. Dhingra, R. Gupta,

A. Kaur, A. Kaur, M. Kaur, S. Kaur, R. Kumar, P. Kumari, M. Lohan, A. Mehta,

K. Sandeep, S. Sharma, J.B. Singh, G. Walia

University of Delhi, Delhi, India

A. Bhardwaj, B.C. Choudhary, R.B. Garg, M. Gola, S. Keshri, Ashok Kumar, S. Malhotra, M. Naimuddin, P. Priyanka, K. Ranjan, Aashaq Shah, R. Sharma

Saha Institute of Nuclear Physics, HBNI, Kolkata, India

R. Bhardwaj ${ }^{24}$, M. Bharti, R. Bhattacharya, S. Bhattacharya, U. Bhawandeep ${ }^{24}$, D. Bhowmik, S. Dey, S. Dutt ${ }^{24}$, S. Dutta, S. Ghosh, K. Mondal, S. Nandan, A. Purohit, P.K. Rout, A. Roy, S. Roy Chowdhury, S. Sarkar, M. Sharan, B. Singh, S. Thakur ${ }^{24}$

Indian Institute of Technology Madras, Madras, India

P.K. Behera

Bhabha Atomic Research Centre, Mumbai, India

R. Chudasama, D. Dutta, V. Jha, V. Kumar, P.K. Netrakanti, L.M. Pant, P. Shukla

Tata Institute of Fundamental Research-A, Mumbai, India

T. Aziz, M.A. Bhat, S. Dugad, G.B. Mohanty, N. Sur, B. Sutar, RavindraKumar Verma

Tata Institute of Fundamental Research-B, Mumbai, India

S. Banerjee, S. Bhattacharya, S. Chatterjee, P. Das, M. Guchait, Sa. Jain, S. Karmakar, S. Kumar, M. Maity ${ }^{25}$, G. Majumder, K. Mazumdar, N. Sahoo, T. Sarkar ${ }^{25}$

Indian Institute of Science Education and Research (IISER), Pune, India

S. Chauhan, S. Dube, V. Hegde, A. Kapoor, K. Kothekar, S. Pandey, A. Rane, S. Sharma

Institute for Research in Fundamental Sciences (IPM), Tehran, Iran

S. Chenarani ${ }^{26}$, E. Eskandari Tadavani, S.M. Etesami ${ }^{26}$, M. Khakzad, M. Mohammadi Najafabadi, M. Naseri, F. Rezaei Hosseinabadi, B. Safarzadeh ${ }^{27}$, M. Zeinali

University College Dublin, Dublin, Ireland

M. Felcini, M. Grunewald

INFN Sezione di Bari ${ }^{a}$, Università di Bari ${ }^{b}$, Politecnico di Bari ${ }^{c}$, Bari, Italy M. Abbrescia ${ }^{a, b}$, C. Calabria ${ }^{a, b}$, A. Colaleo ${ }^{a}$, D. Creanza ${ }^{a, c}$, L. Cristella ${ }^{a, b}$, N. De Filippis $^{a, c}$, M. De Palma ${ }^{a, b}$, A. Di Florio ${ }^{a, b}$, F. Errico ${ }^{a, b}$, L. Fiore ${ }^{a}$, A. Gelmi ${ }^{a, b}$,

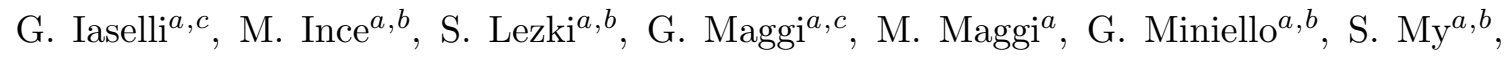
S. Nuzzo ${ }^{a, b}$, A. Pompili ${ }^{a, b}$, G. Pugliese ${ }^{a, c}$, R. Radogna ${ }^{a}$, A. Ranieri ${ }^{a}$, G. Selvaggi ${ }^{a}, b$,

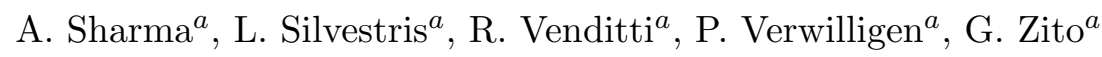

INFN Sezione di Bologna ${ }^{a}$, Università di Bologna ${ }^{b}$, Bologna, Italy

G. Abbiendi ${ }^{a}$, C. Battilana ${ }^{a, b}$, D. Bonacorsi ${ }^{a}, b$, L. Borgonovi $^{a}, b$, S. Braibant-Giacomelli ${ }^{a, b}$,

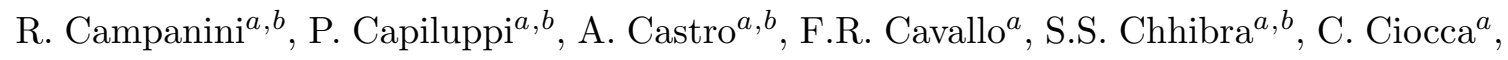

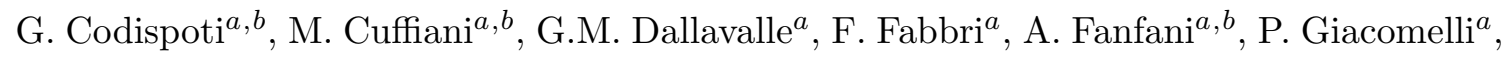

C. Grandi ${ }^{a}$, L. Guiducci ${ }^{a}, b$, F. Iemmi ${ }^{a, b}$, S. Marcellini ${ }^{a}$, G. Masetti ${ }^{a}$, A. Montanari ${ }^{a}$, 


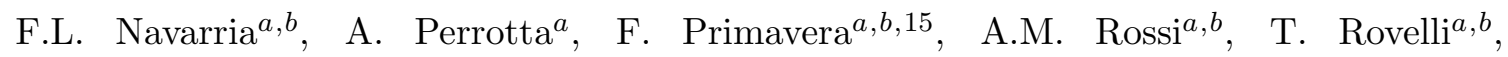
G.P. Siroli ${ }^{a, b}$, N. Tosi ${ }^{a}$

INFN Sezione di Catania ${ }^{a}$, Università di Catania ${ }^{b}$, Catania, Italy

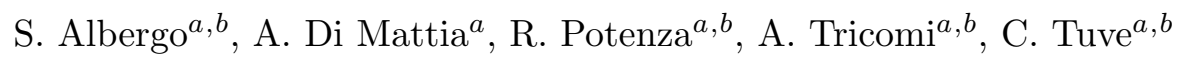

INFN Sezione di Firenze ${ }^{a}$, Università di Firenze ${ }^{b}$, Firenze, Italy

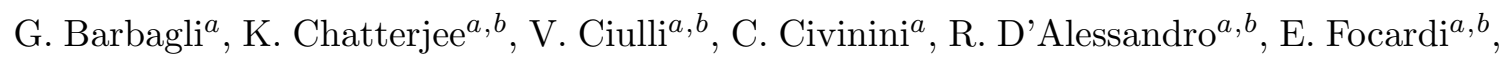

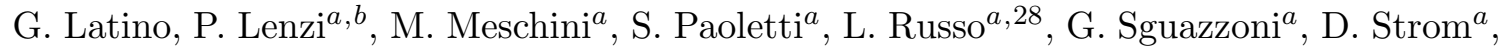

L. Viliani ${ }^{a}$

INFN Laboratori Nazionali di Frascati, Frascati, Italy

L. Benussi, S. Bianco, F. Fabbri, D. Piccolo

INFN Sezione di Genova ${ }^{a}$, Università di Genova ${ }^{b}$, Genova, Italy

F. Ferro ${ }^{a}$, F. Ravera ${ }^{a, b}$, E. Robutti $^{a}$, S. Tosi ${ }^{a, b}$

INFN Sezione di Milano-Bicocca ${ }^{a}$, Università di Milano-Bicocca ${ }^{b}$, Milano, Italy

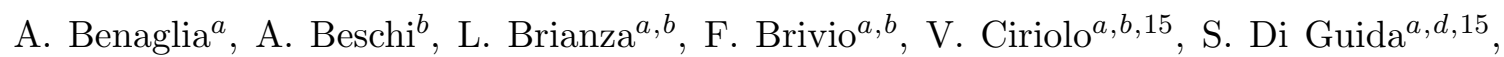
M.E. Dinardo ${ }^{a, b}$, S. Fiorendi ${ }^{a, b}$, S. Gennai ${ }^{a}$, A. Ghezzi ${ }^{a, b}$, P. Govoni ${ }^{a}, b$, M. Malberti ${ }^{a, b}$, S. Malvezzi ${ }^{a}$, A. Massironi ${ }^{a, b}$, D. Menasce ${ }^{a}$, L. Moroni ${ }^{a}$, M. Paganoni ${ }^{a}, b$, D. Pedrini ${ }^{a}$, S. Ragazzi ${ }^{a, b}$, T. Tabarelli de Fatis ${ }^{a, b}$

INFN Sezione di Napoli ${ }^{a}$, Università di Napoli 'Federico II' ${ }^{b}$, Napoli, Italy, Università della Basilicata ${ }^{c}$, Potenza, Italy, Università G. Marconi ${ }^{d}$, Roma, Italy

S. Buontempo ${ }^{a}$, N. Cavallo ${ }^{a, c}$, A. Di Crescenzo ${ }^{a, b}$, F. Fabozzi ${ }^{a, c}$, F. Fienga ${ }^{a}$, G. Galati ${ }^{a}$,

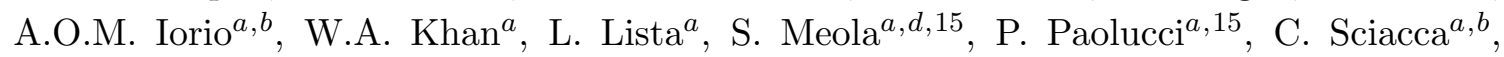
E. Voevodina $a^{a, b}$

INFN Sezione di Padova ${ }^{a}$, Università di Padova ${ }^{b}$, Padova, Italy, Università di Trento ${ }^{c}$, Trento, Italy

P. Azzi ${ }^{a}$, N. Bacchetta ${ }^{a}$, D. Bisello ${ }^{a, b}$, A. Boletti ${ }^{a, b}$, A. Bragagnolo, R. Carlin ${ }^{a, b}$,

P. Checchia ${ }^{a}$, M. Dall'Osso ${ }^{a}, b$, P. De Castro Manzano ${ }^{a}$, T. Dorigo ${ }^{a}$, U. Dosselli ${ }^{a}$, F. Gasparini ${ }^{a, b}$, U. Gasparini ${ }^{a, b}$, A. Gozzelino ${ }^{a}$, S. Lacaprara ${ }^{a}$, P. Lujan, M. Margoni ${ }^{a, b}$, A.T. Meneguzzo ${ }^{a, b}$, J. Pazzini $^{a, b}$, P. Ronchese ${ }^{a, b}$, R. Rossin ${ }^{a, b}$, F. Simonetto ${ }^{a, b}$, A. Tiko, E. Torassa ${ }^{a}$, M. Zanetti ${ }^{a, b}$, P. Zotto ${ }^{a, b}$, G. Zumerle ${ }^{a, b}$

INFN Sezione di Pavia ${ }^{a}$, Università di Pavia ${ }^{b}$, Pavia, Italy

A. Braghieri ${ }^{a}$, A. Magnani ${ }^{a}$, P. Montagna ${ }^{a, b}$, S.P. Ratti ${ }^{a}, b$, V. $\operatorname{Re}^{a}$, M. Ressegotti $^{a, b}$, C. Riccardi ${ }^{a, b}$, P. Salvini ${ }^{a}$, I. Vai ${ }^{a, b}$, P. Vitulo ${ }^{a, b}$

INFN Sezione di Perugia ${ }^{a}$, Università di Perugia ${ }^{b}$, Perugia, Italy

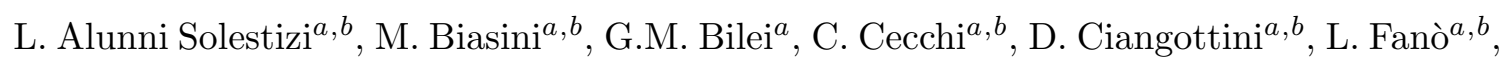

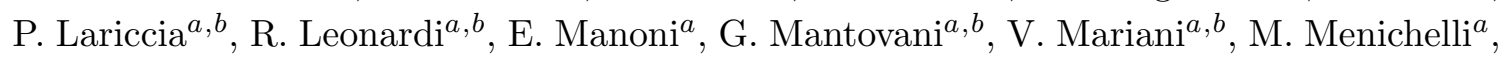
A. Rossi ${ }^{a, b}$, A. Santocchia ${ }^{a, b}$, D. Spiga ${ }^{a}$ 
INFN Sezione di Pisa ${ }^{a}$, Università di Pisa ${ }^{b}$, Scuola Normale Superiore di Pisa ${ }^{c}$, Pisa, Italy

K. Androsov ${ }^{a}$, P. Azzurri ${ }^{a}$, G. Bagliesi ${ }^{a}$, L. Bianchini ${ }^{a}$, T. Boccali ${ }^{a}$, L. Borrello, R. Castaldi ${ }^{a}$, M.A. Ciocci ${ }^{a}, b$, R. Dell'Orso ${ }^{a}$, G. Fedi ${ }^{a}$, F. Fiori ${ }^{a, c}$, L. Giannini $^{a, c}$, A. Giassi $^{a}$, M.T. Grippo ${ }^{a}$, F. Ligabue ${ }^{a, c}$, E. Manca ${ }^{a, c}$, G. Mandorlia ${ }^{a, c}$, A. Messineo ${ }^{a, b}$, F. Palla ${ }^{a}$,

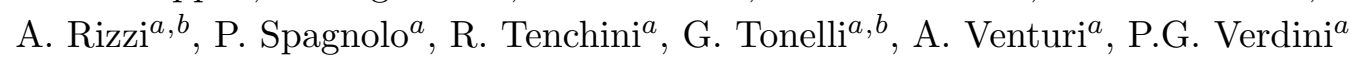

INFN Sezione di Roma ${ }^{a}$, Sapienza Università di Roma ${ }^{b}$, Rome, Italy

L. Barone ${ }^{a, b}$, F. Cavallari ${ }^{a}$, M. Cipriani ${ }^{a, b}$, N. Daci ${ }^{a}$, D. Del Re ${ }^{a, b}$, E. Di Marco ${ }^{a, b}$, M. Diemoz ${ }^{a}$, S. Gelli ${ }^{a, b}$, E. Longo ${ }^{a, b}$, B. Marzocchi ${ }^{a, b}$, P. Meridiani ${ }^{a}$, G. Organtini ${ }^{a, b}$,

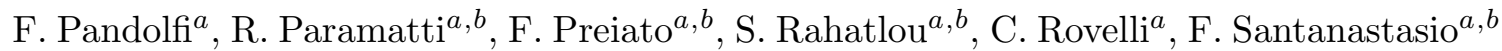

INFN Sezione di Torino ${ }^{a}$, Università di Torino ${ }^{b}$, Torino, Italy, Università del Piemonte Orientale ${ }^{c}$, Novara, Italy

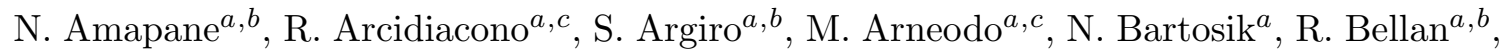

C. Biino ${ }^{a}$, N. Cartiglia $a$, F. Cenna ${ }^{a, b}$, S. Cometti, M. Costa ${ }^{a, b}$, R. Covarelli ${ }^{a, b}$,

N. Demaria ${ }^{a}$, B. Kiani ${ }^{a, b}$, C. Mariotti ${ }^{a}$, S. Maselli ${ }^{a}$, E. Migliore ${ }^{a, b}$, V. Monaco ${ }^{a, b}$,

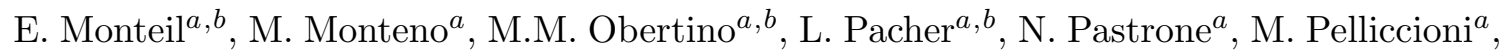
G.L. Pinna Angioni ${ }^{a, b}$, A. Romero ${ }^{a, b}$, M. Ruspa ${ }^{a, c}$, R. Sacchi ${ }^{a, b}$, K. Shchelina ${ }^{a, b}$, V. Sola ${ }^{a}$, A. Solano ${ }^{a, b}$, D. Soldi, A. Staiano ${ }^{a}$

INFN Sezione di Trieste ${ }^{a}$, Università di Trieste ${ }^{b}$, Trieste, Italy

S. Belforte ${ }^{a}$, V. Candelise ${ }^{a, b}$, M. Casarsa ${ }^{a}$, F. Cossutti ${ }^{a}$, G. Della Ricca ${ }^{a, b}$, F. Vazzoler $^{a}, b$, A. Zanetti ${ }^{a}$

Kyungpook National University

D.H. Kim, G.N. Kim, M.S. Kim, J. Lee, S. Lee, S.W. Lee, C.S. Moon, Y.D. Oh, S. Sekmen, D.C. Son, Y.C. Yang

Chonnam National University, Institute for Universe and Elementary Particles, Kwangju, Korea

H. Kim, D.H. Moon, G. Oh

Hanyang University, Seoul, Korea

J. Goh ${ }^{29}$, T.J. Kim

Korea University, Seoul, Korea

S. Cho, S. Choi, Y. Go, D. Gyun, S. Ha, B. Hong, Y. Jo, K. Lee, K.S. Lee, S. Lee, J. Lim, S.K. Park, Y. Roh

Sejong University, Seoul, Korea

H.S. Kim

Seoul National University, Seoul, Korea

J. Almond, J. Kim, J.S. Kim, H. Lee, K. Lee, K. Nam, S.B. Oh, B.C. Radburn-Smith, S.h. Seo, U.K. Yang, H.D. Yoo, G.B. Yu

University of Seoul, Seoul, Korea

D. Jeon, H. Kim, J.H. Kim, J.S.H. Lee, I.C. Park 
Sungkyunkwan University, Suwon, Korea

Y. Choi, C. Hwang, J. Lee, I. Yu

Vilnius University, Vilnius, Lithuania

V. Dudenas, A. Juodagalvis, J. Vaitkus

National Centre for Particle Physics, Universiti Malaya, Kuala Lumpur, Malaysia

I. Ahmed, Z.A. Ibrahim, M.A.B. Md $\mathrm{Ali}^{30}$, F. Mohamad Idris ${ }^{31}$, W.A.T. Wan Abdullah, M.N. Yusli, Z. Zolkapli

Universidad de Sonora (UNISON), Hermosillo, Mexico

A. Castaneda Hernandez, J.A. Murillo Quijada

Centro de Investigacion y de Estudios Avanzados del IPN, Mexico City, Mexico

H. Castilla-Valdez, E. De La Cruz-Burelo, M.C. Duran-Osuna, I. Heredia-De La Cruz ${ }^{32}$, R. Lopez-Fernandez, J. Mejia Guisao, R.I. Rabadan-Trejo, G. Ramirez-Sanchez, R ReyesAlmanza, A. Sanchez-Hernandez

Universidad Iberoamericana, Mexico City, Mexico

S. Carrillo Moreno, C. Oropeza Barrera, F. Vazquez Valencia

Benemerita Universidad Autonoma de Puebla, Puebla, Mexico

J. Eysermans, I. Pedraza, H.A. Salazar Ibarguen, C. Uribe Estrada

Universidad Autónoma de San Luis Potosí, San Luis Potosí, Mexico

A. Morelos Pineda

University of Auckland, Auckland, New Zealand

D. Krofcheck

University of Canterbury, Christchurch, New Zealand

S. Bheesette, P.H. Butler

National Centre for Physics, Quaid-I-Azam University, Islamabad, Pakistan

A. Ahmad, M. Ahmad, M.I. Asghar, Q. Hassan, H.R. Hoorani, A. Saddique, M.A. Shah, M. Shoaib, M. Waqas

National Centre for Nuclear Research, Swierk, Poland

H. Bialkowska, M. Bluj, B. Boimska, T. Frueboes, M. Górski, M. Kazana, K. Nawrocki, M. Szleper, P. Traczyk, P. Zalewski

Institute of Experimental Physics, Faculty of Physics, University of Warsaw, Warsaw, Poland

K. Bunkowski, A. Byszuk ${ }^{33}$, K. Doroba, A. Kalinowski, M. Konecki, J. Krolikowski, M. Misiura, M. Olszewski, A. Pyskir, M. Walczak 
Laboratório de Instrumentação e Física Experimental de Partículas, Lisboa, Portugal

P. Bargassa, C. Beirão Da Cruz E Silva, A. Di Francesco, P. Faccioli, B. Galinhas, M. Gallinaro, J. Hollar, N. Leonardo, L. Lloret Iglesias, M.V. Nemallapudi, J. Seixas, G. Strong, O. Toldaiev, D. Vadruccio, J. Varela

\section{Joint Institute for Nuclear Research, Dubna, Russia}

S. Afanasiev, V. Alexakhin, P. Bunin, M. Gavrilenko, A. Golunov, I. Golutvin, N. Gorbounov, V. Karjavin, A. Lanev, A. Malakhov, V. Matveev ${ }^{34,35}$, P. Moisenz, V. Palichik, V. Perelygin, M. Savina, S. Shmatov, V. Smirnov, N. Voytishin, A. Zarubin

Petersburg Nuclear Physics Institute, Gatchina (St. Petersburg), Russia

V. Golovtsov, Y. Ivanov, V. Kim ${ }^{36}$, E. Kuznetsova ${ }^{37}$, P. Levchenko, V. Murzin, V. Oreshkin, I. Smirnov, D. Sosnov, V. Sulimov, L. Uvarov, S. Vavilov, A. Vorobyev

Institute for Nuclear Research, Moscow, Russia

Yu. Andreev, A. Dermenev, S. Gninenko, N. Golubev, A. Karneyeu, M. Kirsanov, N. Krasnikov, A. Pashenkov, D. Tlisov, A. Toropin

Institute for Theoretical and Experimental Physics, Moscow, Russia

V. Epshteyn, V. Gavrilov, N. Lychkovskaya, V. Popov, I. Pozdnyakov, G. Safronov, A. Spiridonov, A. Stepennov, V. Stolin, M. Toms, E. Vlasov, A. Zhokin

Moscow Institute of Physics and Technology, Moscow, Russia

T. Aushev

National Research Nuclear University 'Moscow Engineering Physics Institute' (MEPhI), Moscow, Russia

R. Chistov ${ }^{38}$, M. Danilov ${ }^{38}$, P. Parygin, D. Philippov, S. Polikarpov ${ }^{38}$, E. Tarkovskii

P.N. Lebedev Physical Institute, Moscow, Russia

V. Andreev, M. Azarkin ${ }^{35}$, I. Dremin ${ }^{35}$, M. Kirakosyan ${ }^{35}$, S.V. Rusakov, A. Terkulov

Skobeltsyn Institute of Nuclear Physics, Lomonosov Moscow State University, Moscow, Russia

A. Baskakov, A. Belyaev, E. Boos, V. Bunichev, M. Dubinin ${ }^{39}$, L. Dudko, A. Ershov,

A. Gribushin, V. Klyukhin, I. Lokhtin, I. Miagkov, S. Obraztsov, S. Petrushanko, V. Savrin,

A. Snigirev

Novosibirsk State University (NSU), Novosibirsk, Russia

V. Blinov ${ }^{40}$, T. Dimova ${ }^{40}$, L. Kardapoltsev ${ }^{40}$, D. Shtol ${ }^{40}$, Y. Skovpen ${ }^{40}$

State Research Center of Russian Federation, Institute for High Energy Physics of NRC "Kurchatov Institute", Protvino, Russia

I. Azhgirey, I. Bayshev, S. Bitioukov, D. Elumakhov, A. Godizov, V. Kachanov, A. Kalinin,

D. Konstantinov, P. Mandrik, V. Petrov, R. Ryutin, S. Slabospitskii, A. Sobol, S. Troshin,

N. Tyurin, A. Uzunian, A. Volkov 
National Research Tomsk Polytechnic University, Tomsk, Russia

A. Babaev, S. Baidali

University of Belgrade, Faculty of Physics and Vinca Institute of Nuclear Sciences, Belgrade, Serbia

P. Adzic ${ }^{41}$, P. Cirkovic, D. Devetak, M. Dordevic, J. Milosevic

Centro de Investigaciones Energéticas Medioambientales y Tecnológicas (CIEMAT), Madrid, Spain

J. Alcaraz Maestre, A. Álvarez Fernández, I. Bachiller, M. Barrio Luna, J.A. Brochero Cifuentes, M. Cerrada, N. Colino, B. De La Cruz, A. Delgado Peris, C. Fernandez Bedoya, J.P. Fernández Ramos, J. Flix, M.C. Fouz, O. Gonzalez Lopez, S. Goy Lopez, J.M. Hernandez, M.I. Josa, D. Moran, A. Pérez-Calero Yzquierdo, J. Puerta Pelayo, I. Redondo, L. Romero, M.S. Soares, A. Triossi

Universidad Autónoma de Madrid, Madrid, Spain

C. Albajar, J.F. de Trocóniz

Universidad de Oviedo, Oviedo, Spain

J. Cuevas, C. Erice, J. Fernandez Menendez, S. Folgueras, I. Gonzalez Caballero, J.R. González Fernández, E. Palencia Cortezon, V. Rodríguez Bouza, S. Sanchez Cruz, P. Vischia, J.M. Vizan Garcia

Instituto de Física de Cantabria (IFCA), CSIC-Universidad de Cantabria, Santander, Spain

I.J. Cabrillo, A. Calderon, B. Chazin Quero, J. Duarte Campderros, M. Fernandez, P.J. Fernández Manteca, A. García Alonso, J. Garcia-Ferrero, G. Gomez, A. Lopez Virto, J. Marco, C. Martinez Rivero, P. Martinez Ruiz del Arbol, F. Matorras, J. Piedra Gomez, C. Prieels, T. Rodrigo, A. Ruiz-Jimeno, L. Scodellaro, N. Trevisani, I. Vila, R. Vilar Cortabitarte

CERN, European Organization for Nuclear Research, Geneva, Switzerland

D. Abbaneo, B. Akgun, E. Auffray, P. Baillon, A.H. Ball, D. Barney, J. Bendavid, M. Bianco, A. Bocci, C. Botta, E. Brondolin, T. Camporesi, M. Cepeda, G. Cerminara, E. Chapon, Y. Chen, G. Cucciati, D. d'Enterria, A. Dabrowski, V. Daponte, A. David, A. De Roeck, N. Deelen, M. Dobson, T. du Pree, M. Dünser, N. Dupont, A. ElliottPeisert, P. Everaerts, F. Fallavollita ${ }^{42}$, D. Fasanella, G. Franzoni, J. Fulcher, W. Funk, D. Gigi, A. Gilbert, K. Gill, F. Glege, M. Guilbaud, D. Gulhan, J. Hegeman, V. Innocente, A. Jafari, P. Janot, O. Karacheban ${ }^{18}$, J. Kieseler, A. Kornmayer, M. Krammer ${ }^{1}$, C. Lange, P. Lecoq, C. Lourenço, L. Malgeri, M. Mannelli, F. Meijers, J.A. Merlin, S. Mersi, E. Meschi, P. Milenovic ${ }^{43}$, F. Moortgat, M. Mulders, J. Ngadiuba, S. Orfanelli, L. Orsini, F. Pantaleo ${ }^{15}$, L. Pape, E. Perez, M. Peruzzi, A. Petrilli, G. Petrucciani, A. Pfeiffer, M. Pierini, F.M. Pitters, D. Rabady, A. Racz, T. Reis, G. Rolandi ${ }^{44}$, M. Rovere, H. Sakulin, C. Schäfer, C. Schwick, M. Seidel, M. Selvaggi, A. Sharma, P. Silva, P. Sphicas ${ }^{45}$, A. Stakia, J. Steggemann, M. Tosi, D. Treille, A. Tsirou, V. Veckalns ${ }^{46}$, W.D. Zeuner 
Paul Scherrer Institut, Villigen, Switzerland

L. Caminada ${ }^{47}$, K. Deiters, W. Erdmann, R. Horisberger, Q. Ingram, H.C. Kaestli, D. Kotlinski, U. Langenegger, T. Rohe, S.A. Wiederkehr

ETH Zurich - Institute for Particle Physics and Astrophysics (IPA), Zurich, Switzerland

M. Backhaus, L. Bäni, P. Berger, N. Chernyavskaya, G. Dissertori, M. Dittmar, M. Donegà, C. Dorfer, C. Grab, C. Heidegger, D. Hits, J. Hoss, T. Klijnsma, W. Lustermann, R.A. Manzoni, M. Marionneau, M.T. Meinhard, F. Micheli, P. Musella, F. Nessi-Tedaldi, J. Pata, F. Pauss, G. Perrin, L. Perrozzi, S. Pigazzini, M. Quittnat, D. Ruini, D.A. Sanz Becerra, M. Schönenberger, L. Shchutska, V.R. Tavolaro, K. Theofilatos, M.L. Vesterbacka Olsson, R. Wallny, D.H. Zhu

Universität Zürich, Zurich, Switzerland

T.K. Aarrestad, C. Amsler ${ }^{48}$, D. Brzhechko, M.F. Canelli, A. De Cosa, R. Del Burgo, S. Donato, C. Galloni, T. Hreus, B. Kilminster, I. Neutelings, D. Pinna, G. Rauco, P. Robmann, D. Salerno, K. Schweiger, C. Seitz, Y. Takahashi, A. Zucchetta

\section{National Central University, Chung-Li, Taiwan}

Y.H. Chang, K.y. Cheng, T.H. Doan, Sh. Jain, R. Khurana, C.M. Kuo, W. Lin, A. Pozdnyakov, S.S. Yu

\section{National Taiwan University (NTU), Taipei, Taiwan}

P. Chang, Y. Chao, K.F. Chen, P.H. Chen, W.-S. Hou, Arun Kumar, Y.y. Li, Y.F. Liu, R.-S. Lu, E. Paganis, A. Psallidas, A. Steen, J.f. Tsai

Chulalongkorn University, Faculty of Science, Department of Physics, Bangkok, Thailand

B. Asavapibhop, N. Srimanobhas, N. Suwonjandee

Çukurova University, Physics Department, Science and Art Faculty, Adana, Turkey

A. Bat, F. Boran, S. Cerci ${ }^{49}$, S. Damarseckin, Z.S. Demiroglu, F. Dolek, C. Dozen, I. Dumanoglu, S. Girgis, G. Gokbulut, Y. Guler, E. Gurpinar, I. Hos ${ }^{50}$, C. Isik, E.E. Kangal ${ }^{51}$, O. Kara, A. Kayis Topaksu, U. Kiminsu, M. Oglakci, G. Onengut, K. Ozdemir ${ }^{52}$, S. Ozturk ${ }^{53}$, D. Sunar Cerci ${ }^{49}$, B. Tali ${ }^{49}$, U.G. Tok, S. Turkcapar, I.S. Zorbakir, C. Zorbilmez

Middle East Technical University, Physics Department, Ankara, Turkey

B. Isildak ${ }^{54}$, G. Karapinar ${ }^{55}$, M. Yalvac, M. Zeyrek

Bogazici University, Istanbul, Turkey

I.O. Atakisi, E. Gülmez, M. Kaya ${ }^{56}$, O. Kaya ${ }^{57}$, S. Tekten, E.A. Yetkin ${ }^{58}$

Istanbul Technical University, Istanbul, Turkey

M.N. Agaras, S. Atay, A. Cakir, K. Cankocak, Y. Komurcu, S. Sen ${ }^{59}$ 
Institute for Scintillation Materials of National Academy of Science of Ukraine, Kharkov, Ukraine

B. Grynyov

National Scientific Center, Kharkov Institute of Physics and Technology, Kharkov, Ukraine

L. Levchuk

University of Bristol, Bristol, United Kingdom

F. Ball, L. Beck, J.J. Brooke, D. Burns, E. Clement, D. Cussans, O. Davignon, H. Flacher, J. Goldstein, G.P. Heath, H.F. Heath, L. Kreczko, D.M. Newbold ${ }^{60}$, S. Paramesvaran, B. Penning, T. Sakuma, D. Smith, V.J. Smith, J. Taylor, A. Titterton

\section{Rutherford Appleton Laboratory, Didcot, United Kingdom}

K.W. Bell, A. Belyaev ${ }^{61}$, C. Brew, R.M. Brown, D. Cieri, D.J.A. Cockerill, J.A. Coughlan, K. Harder, S. Harper, J. Linacre, E. Olaiya, D. Petyt, C.H. Shepherd-Themistocleous, A. Thea, I.R. Tomalin, T. Williams, W.J. Womersley

\section{Imperial College, London, United Kingdom}

G. Auzinger, R. Bainbridge, P. Bloch, J. Borg, S. Breeze, O. Buchmuller, A. Bundock, S. Casasso, D. Colling, L. Corpe, P. Dauncey, G. Davies, M. Della Negra, R. Di Maria, Y. Haddad, G. Hall, G. Iles, T. James, M. Komm, C. Laner, L. Lyons, A.-M. Magnan, S. Malik, A. Martelli, J. Nash ${ }^{62}$, A. Nikitenko ${ }^{7}$, V. Palladino, M. Pesaresi, A. Richards, A. Rose, E. Scott, C. Seez, A. Shtipliyski, G. Singh, M. Stoye, T. Strebler, S. Summers, A. Tapper, K. Uchida, T. Virdee ${ }^{15}$, N. Wardle, D. Winterbottom, J. Wright, S.C. Zenz

Brunel University, Uxbridge, United Kingdom

J.E. Cole, P.R. Hobson, A. Khan, P. Kyberd, C.K. Mackay, A. Morton, I.D. Reid, L. Teodorescu, S. Zahid

Baylor University, Waco, U.S.A.

K. Call, J. Dittmann, K. Hatakeyama, H. Liu, C. Madrid, B. Mcmaster, N. Pastika, C. Smith

Catholic University of America, Washington DC, U.S.A.

R. Bartek, A. Dominguez

The University of Alabama, Tuscaloosa, U.S.A.

A. Buccilli, S.I. Cooper, C. Henderson, P. Rumerio, C. West

Boston University, Boston, U.S.A.

D. Arcaro, T. Bose, D. Gastler, D. Rankin, C. Richardson, J. Rohlf, L. Sulak, D. Zou

Brown University, Providence, U.S.A.

G. Benelli, X. Coubez, D. Cutts, M. Hadley, J. Hakala, U. Heintz, J.M. Hogan ${ }^{63}$, K.H.M. Kwok, E. Laird, G. Landsberg, J. Lee, Z. Mao, M. Narain, S. Piperov, S. Sagir ${ }^{64}$, R. Syarif, E. Usai, D. Yu 
University of California, Davis, Davis, U.S.A.

R. Band, C. Brainerd, R. Breedon, D. Burns, M. Calderon De La Barca Sanchez,

M. Chertok, J. Conway, R. Conway, P.T. Cox, R. Erbacher, C. Flores, G. Funk, W. Ko,

O. Kukral, R. Lander, C. Mclean, M. Mulhearn, D. Pellett, J. Pilot, S. Shalhout, M. Shi,

D. Stolp, D. Taylor, K. Tos, M. Tripathi, Z. Wang, F. Zhang

University of California, Los Angeles, U.S.A.

M. Bachtis, C. Bravo, R. Cousins, A. Dasgupta, A. Florent, J. Hauser, M. Ignatenko, N. Mccoll, S. Regnard, D. Saltzberg, C. Schnaible, V. Valuev

University of California, Riverside, Riverside, U.S.A.

E. Bouvier, K. Burt, R. Clare, J.W. Gary, S.M.A. Ghiasi Shirazi, G. Hanson, G. Karapostoli, E. Kennedy, F. Lacroix, O.R. Long, M. Olmedo Negrete, M.I. Paneva, W. Si, L. Wang, H. Wei, S. Wimpenny, B.R. Yates

University of California, San Diego, La Jolla, U.S.A.

J.G. Branson, S. Cittolin, M. Derdzinski, R. Gerosa, D. Gilbert, B. Hashemi, A. Holzner, D. Klein, G. Kole, V. Krutelyov, J. Letts, M. Masciovecchio, D. Olivito, S. Padhi, M. Pieri, M. Sani, V. Sharma, S. Simon, M. Tadel, A. Vartak, S. Wasserbaech ${ }^{65}$, J. Wood, F. Würthwein, A. Yagil, G. Zevi Della Porta

University of California, Santa Barbara - Department of Physics, Santa Barbara, U.S.A.

N. Amin, R. Bhandari, J. Bradmiller-Feld, C. Campagnari, M. Citron, A. Dishaw, V. Dutta, M. Franco Sevilla, L. Gouskos, R. Heller, J. Incandela, A. Ovcharova, H. Qu, J. Richman, D. Stuart, I. Suarez, S. Wang, J. Yoo

California Institute of Technology, Pasadena, U.S.A.

D. Anderson, A. Bornheim, J.M. Lawhorn, H.B. Newman, T.Q. Nguyen, M. Spiropulu, J.R. Vlimant, R. Wilkinson, S. Xie, Z. Zhang, R.Y. Zhu

Carnegie Mellon University, Pittsburgh, U.S.A.

M.B. Andrews, T. Ferguson, T. Mudholkar, M. Paulini, M. Sun, I. Vorobiev, M. Weinberg

University of Colorado Boulder, Boulder, U.S.A.

J.P. Cumalat, W.T. Ford, F. Jensen, A. Johnson, M. Krohn, S. Leontsinis, E. MacDonald, T. Mulholland, K. Stenson, K.A. Ulmer, S.R. Wagner

Cornell University, Ithaca, U.S.A.

J. Alexander, J. Chaves, Y. Cheng, J. Chu, A. Datta, K. Mcdermott, N. Mirman, J.R. Patterson, D. Quach, A. Rinkevicius, A. Ryd, L. Skinnari, L. Soffi, S.M. Tan, Z. Tao, J. Thom, J. Tucker, P. Wittich, M. Zientek

Fermi National Accelerator Laboratory, Batavia, U.S.A.

S. Abdullin, M. Albrow, M. Alyari, G. Apollinari, A. Apresyan, A. Apyan, S. Banerjee, L.A.T. Bauerdick, A. Beretvas, J. Berryhill, P.C. Bhat, G. Bolla ${ }^{\dagger}$, K. Burkett, J.N. Butler, A. Canepa, G.B. Cerati, H.W.K. Cheung, F. Chlebana, M. Cremonesi, J. Duarte, V.D. Elvira, J. Freeman, Z. Gecse, E. Gottschalk, L. Gray, D. Green, S. Grünendahl, 
O. Gutsche, J. Hanlon, R.M. Harris, S. Hasegawa, J. Hirschauer, Z. Hu, B. Jayatilaka, S. Jindariani, M. Johnson, U. Joshi, B. Klima, M.J. Kortelainen, B. Kreis, S. Lammel, D. Lincoln, R. Lipton, M. Liu, T. Liu, J. Lykken, K. Maeshima, J.M. Marraffino, D. Mason, P. McBride, P. Merkel, S. Mrenna, S. Nahn, V. O’Dell, K. Pedro, C. Pena, O. Prokofyev, G. Rakness, L. Ristori, A. Savoy-Navarro ${ }^{66}$, B. Schneider, E. Sexton-Kennedy, A. Soha, W.J. Spalding, L. Spiegel, S. Stoynev, J. Strait, N. Strobbe, L. Taylor, S. Tkaczyk, N.V. Tran, L. Uplegger, E.W. Vaandering, C. Vernieri, M. Verzocchi, R. Vidal, M. Wang, H.A. Weber, A. Whitbeck

University of Florida, Gainesville, U.S.A.

D. Acosta, P. Avery, P. Bortignon, D. Bourilkov, A. Brinkerhoff, L. Cadamuro, A. Carnes, M. Carver, D. Curry, R.D. Field, S.V. Gleyzer, B.M. Joshi, J. Konigsberg, A. Korytov, P. Ma, K. Matchev, H. Mei, G. Mitselmakher, K. Shi, D. Sperka, J. Wang, S. Wang

Florida International University, Miami, U.S.A.

Y.R. Joshi, S. Linn

Florida State University, Tallahassee, U.S.A.

A. Ackert, T. Adams, A. Askew, S. Hagopian, V. Hagopian, K.F. Johnson, T. Kolberg, G. Martinez, T. Perry, H. Prosper, A. Saha, V. Sharma, R. Yohay

Florida Institute of Technology, Melbourne, U.S.A.

M.M. Baarmand, V. Bhopatkar, S. Colafranceschi, M. Hohlmann, D. Noonan, M. Rahmani, T. Roy, F. Yumiceva

\section{University of Illinois at Chicago (UIC), Chicago, U.S.A.}

M.R. Adams, L. Apanasevich, D. Berry, R.R. Betts, R. Cavanaugh, X. Chen, S. Dittmer, O. Evdokimov, C.E. Gerber, D.A. Hangal, D.J. Hofman, K. Jung, J. Kamin, C. Mills, I.D. Sandoval Gonzalez, M.B. Tonjes, N. Varelas, H. Wang, X. Wang, Z. Wu, J. Zhang

The University of Iowa, Iowa City, U.S.A.

M. Alhusseini, B. Bilki ${ }^{67}$, W. Clarida, K. Dilsiz ${ }^{68}$, S. Durgut, R.P. Gandrajula, M. Haytmyradov, V. Khristenko, J.-P. Merlo, A. Mestvirishvili, A. Moeller, J. Nachtman, H. Ogul ${ }^{69}$, Y. Onel, F. Ozok ${ }^{70}$, A. Penzo, C. Snyder, E. Tiras, J. Wetzel

Johns Hopkins University, Baltimore, U.S.A.

B. Blumenfeld, A. Cocoros, N. Eminizer, D. Fehling, L. Feng, A.V. Gritsan, W.T. Hung, P. Maksimovic, J. Roskes, U. Sarica, M. Swartz, M. Xiao, C. You

The University of Kansas, Lawrence, U.S.A.

A. Al-bataineh, P. Baringer, A. Bean, S. Boren, J. Bowen, A. Bylinkin, J. Castle, S. Khalil, A. Kropivnitskaya, D. Majumder, W. Mcbrayer, M. Murray, C. Rogan, S. Sanders, E. Schmitz, J.D. Tapia Takaki, Q. Wang

Kansas State University, Manhattan, U.S.A.

S. Duric, A. Ivanov, K. Kaadze, D. Kim, Y. Maravin, D.R. Mendis, T. Mitchell, A. Modak, A. Mohammadi, L.K. Saini, N. Skhirtladze 
Lawrence Livermore National Laboratory, Livermore, U.S.A.

F. Rebassoo, D. Wright

University of Maryland, College Park, U.S.A.

A. Baden, O. Baron, A. Belloni, S.C. Eno, Y. Feng, C. Ferraioli, N.J. Hadley, S. Jabeen, G.Y. Jeng, R.G. Kellogg, J. Kunkle, A.C. Mignerey, F. Ricci-Tam, Y.H. Shin, A. Skuja, S.C. Tonwar, K. Wong

Massachusetts Institute of Technology, Cambridge, U.S.A.

D. Abercrombie, B. Allen, V. Azzolini, A. Baty, G. Bauer, R. Bi, S. Brandt, W. Busza, I.A. Cali, M. D'Alfonso, Z. Demiragli, G. Gomez Ceballos, M. Goncharov, P. Harris, D. Hsu, M. Hu, Y. Iiyama, G.M. Innocenti, M. Klute, D. Kovalskyi, Y.-J. Lee, P.D. Luckey, B. Maier, A.C. Marini, C. Mcginn, C. Mironov, S. Narayanan, X. Niu, C. Paus, C. Roland, G. Roland, G.S.F. Stephans, K. Sumorok, K. Tatar, D. Velicanu, J. Wang, T.W. Wang, B. Wyslouch, S. Zhaozhong

University of Minnesota, Minneapolis, U.S.A.

A.C. Benvenuti, R.M. Chatterjee, A. Evans, P. Hansen, S. Kalafut, Y. Kubota, Z. Lesko, J. Mans, S. Nourbakhsh, N. Ruckstuhl, R. Rusack, J. Turkewitz, M.A. Wadud

University of Mississippi, Oxford, U.S.A.

J.G. Acosta, S. Oliveros

University of Nebraska-Lincoln, Lincoln, U.S.A.

E. Avdeeva, K. Bloom, D.R. Claes, C. Fangmeier, F. Golf, R. Gonzalez Suarez, R. Kamalieddin, I. Kravchenko, J. Monroy, J.E. Siado, G.R. Snow, B. Stieger

State University of New York at Buffalo, Buffalo, U.S.A.

A. Godshalk, C. Harrington, I. Iashvili, A. Kharchilava, D. Nguyen, A. Parker, S. Rappoccio, B. Roozbahani

Northeastern University, Boston, U.S.A.

G. Alverson, E. Barberis, C. Freer, A. Hortiangtham, D.M. Morse, T. Orimoto, R. Teixeira De Lima, T. Wamorkar, B. Wang, A. Wisecarver, D. Wood

Northwestern University, Evanston, U.S.A.

S. Bhattacharya, O. Charaf, K.A. Hahn, N. Mucia, N. Odell, M.H. Schmitt, K. Sung, M. Trovato, M. Velasco

University of Notre Dame, Notre Dame, U.S.A.

R. Bucci, N. Dev, M. Hildreth, K. Hurtado Anampa, C. Jessop, D.J. Karmgard, N. Kellams, K. Lannon, W. Li, N. Loukas, N. Marinelli, F. Meng, C. Mueller, Y. Musienko ${ }^{34}$, M. Planer, A. Reinsvold, R. Ruchti, P. Siddireddy, G. Smith, S. Taroni, M. Wayne, A. Wightman, M. Wolf, A. Woodard

The Ohio State University, Columbus, U.S.A.

J. Alimena, L. Antonelli, B. Bylsma, L.S. Durkin, S. Flowers, B. Francis, A. Hart, C. Hill, W. Ji, T.Y. Ling, W. Luo, B.L. Winer, H.W. Wulsin 
Princeton University, Princeton, U.S.A.

S. Cooperstein, P. Elmer, J. Hardenbrook, P. Hebda, S. Higginbotham, A. Kalogeropoulos,

D. Lange, M.T. Lucchini, J. Luo, D. Marlow, K. Mei, I. Ojalvo, J. Olsen, C. Palmer, P. Piroué, J. Salfeld-Nebgen, D. Stickland, C. Tully

University of Puerto Rico, Mayaguez, U.S.A.

S. Malik, S. Norberg

Purdue University, West Lafayette, U.S.A.

A. Barker, V.E. Barnes, S. Das, L. Gutay, M. Jones, A.W. Jung, A. Khatiwada, B. Mahakud, D.H. Miller, N. Neumeister, C.C. Peng, H. Qiu, J.F. Schulte, J. Sun, F. Wang, R. Xiao, W. Xie

Purdue University Northwest, Hammond, U.S.A.

T. Cheng, J. Dolen, N. Parashar

Rice University, Houston, U.S.A.

Z. Chen, K.M. Ecklund, S. Freed, F.J.M. Geurts, M. Kilpatrick, W. Li, B. Michlin, B.P. Padley, J. Roberts, J. Rorie, W. Shi, Z. Tu, J. Zabel, A. Zhang

University of Rochester, Rochester, U.S.A.

A. Bodek, P. de Barbaro, R. Demina, Y.t. Duh, J.L. Dulemba, C. Fallon, T. Ferbel, M. Galanti, A. Garcia-Bellido, J. Han, O. Hindrichs, A. Khukhunaishvili, K.H. Lo, P. Tan, R. Taus, M. Verzetti

Rutgers, The State University of New Jersey, Piscataway, U.S.A.

A. Agapitos, J.P. Chou, Y. Gershtein, T.A. Gómez Espinosa, E. Halkiadakis, M. Heindl, E. Hughes, S. Kaplan, R. Kunnawalkam Elayavalli, S. Kyriacou, A. Lath, R. Montalvo, K. Nash, M. Osherson, H. Saka, S. Salur, S. Schnetzer, D. Sheffield, S. Somalwar, R. Stone, S. Thomas, P. Thomassen, M. Walker

University of Tennessee, Knoxville, U.S.A.

A.G. Delannoy, J. Heideman, G. Riley, K. Rose, S. Spanier, K. Thapa

Texas A\&M University, College Station, U.S.A.

O. Bouhali ${ }^{71}$, A. Celik, M. Dalchenko, M. De Mattia, A. Delgado, S. Dildick, R. Eusebi, J. Gilmore, T. Huang, T. Kamon ${ }^{72}$, S. Luo, R. Mueller, Y. Pakhotin, R. Patel, A. Perloff, L. Perniè, D. Rathjens, A. Safonov, A. Tatarinov

\section{Texas Tech University, Lubbock, U.S.A.}

N. Akchurin, J. Damgov, F. De Guio, P.R. Dudero, S. Kunori, K. Lamichhane, S.W. Lee, T. Mengke, S. Muthumuni, T. Peltola, S. Undleeb, I. Volobouev, Z. Wang

Vanderbilt University, Nashville, U.S.A.

S. Greene, A. Gurrola, R. Janjam, W. Johns, C. Maguire, A. Melo, H. Ni, K. Padeken, J.D. Ruiz Alvarez, P. Sheldon, S. Tuo, J. Velkovska, M. Verweij, Q. Xu 


\section{University of Virginia, Charlottesville, U.S.A.}

M.W. Arenton, P. Barria, B. Cox, R. Hirosky, M. Joyce, A. Ledovskoy, H. Li, C. Neu, T. Sinthuprasith, Y. Wang, E. Wolfe, F. Xia

\section{Wayne State University, Detroit, U.S.A.}

R. Harr, P.E. Karchin, N. Poudyal, J. Sturdy, P. Thapa, S. Zaleski

University of Wisconsin - Madison, Madison, WI, U.S.A.

M. Brodski, J. Buchanan, C. Caillol, D. Carlsmith, S. Dasu, L. Dodd, B. Gomber, M. Grothe, M. Herndon, A. Hervé, U. Hussain, P. Klabbers, A. Lanaro, A. Levine, K. Long, R. Loveless, T. Ruggles, A. Savin, N. Smith, W.H. Smith, N. Woods

\section{†: Deceased}

1: Also at Vienna University of Technology, Vienna, Austria

2: Also at IRFU, CEA, Université Paris-Saclay, Gif-sur-Yvette, France

3: Also at Universidade Estadual de Campinas, Campinas, Brazil

4: Also at Federal University of Rio Grande do Sul, Porto Alegre, Brazil

5: Also at Université Libre de Bruxelles, Bruxelles, Belgium

6: Also at University of Chinese Academy of Sciences, Beijing, China

7: Also at Institute for Theoretical and Experimental Physics, Moscow, Russia

8: Also at Joint Institute for Nuclear Research, Dubna, Russia

9: Also at Suez University, Suez, Egypt

10: Now at British University in Egypt, Cairo, Egypt

11: Also at Zewail City of Science and Technology, Zewail, Egypt

12: Also at Department of Physics, King Abdulaziz University, Jeddah, Saudi Arabia

13: Also at Université de Haute Alsace, Mulhouse, France

14: Also at Skobeltsyn Institute of Nuclear Physics, Lomonosov Moscow State University, Moscow, Russia

15: Also at CERN, European Organization for Nuclear Research, Geneva, Switzerland

16: Also at RWTH Aachen University, III. Physikalisches Institut A, Aachen, Germany

17: Also at University of Hamburg, Hamburg, Germany

18: Also at Brandenburg University of Technology, Cottbus, Germany

19: Also at MTA-ELTE Lendület CMS Particle and Nuclear Physics Group, Eötvös Loránd University, Budapest, Hungary

20: Also at Institute of Nuclear Research ATOMKI, Debrecen, Hungary

21: Also at Institute of Physics, University of Debrecen, Debrecen, Hungary

22: Also at Indian Institute of Technology Bhubaneswar, Bhubaneswar, India

23: Also at Institute of Physics, Bhubaneswar, India

24: Also at Shoolini University, Solan, India

25: Also at University of Visva-Bharati, Santiniketan, India

26: Also at Isfahan University of Technology, Isfahan, Iran

27: Also at Plasma Physics Research Center, Science and Research Branch, Islamic Azad University, Tehran, Iran

28: Also at Università degli Studi di Siena, Siena, Italy

29: Also at Kyunghee University, Seoul, Korea

30: Also at International Islamic University of Malaysia, Kuala Lumpur, Malaysia

31: Also at Malaysian Nuclear Agency, MOSTI, Kajang, Malaysia

32: Also at Consejo Nacional de Ciencia y Tecnología, Mexico city, Mexico 
33: Also at Warsaw University of Technology, Institute of Electronic Systems, Warsaw, Poland

34: Also at Institute for Nuclear Research, Moscow, Russia

35: Now at National Research Nuclear University 'Moscow Engineering Physics Institute' (MEPhI), Moscow, Russia

36: Also at St. Petersburg State Polytechnical University, St. Petersburg, Russia

37: Also at University of Florida, Gainesville, U.S.A.

38: Also at P.N. Lebedev Physical Institute, Moscow, Russia

39: Also at California Institute of Technology, Pasadena, U.S.A.

40: Also at Budker Institute of Nuclear Physics, Novosibirsk, Russia

41: Also at Faculty of Physics, University of Belgrade, Belgrade, Serbia

42: Also at INFN Sezione di Pavia ${ }^{a}$, Università di Pavia ${ }^{b}$, Pavia, Italy

43: Also at University of Belgrade, Faculty of Physics and Vinca Institute of Nuclear Sciences, Belgrade, Serbia

44: Also at Scuola Normale e Sezione dell'INFN, Pisa, Italy

45: Also at National and Kapodistrian University of Athens, Athens, Greece

46: Also at Riga Technical University, Riga, Latvia

47: Also at Universität Zürich, Zurich, Switzerland

48: Also at Stefan Meyer Institute for Subatomic Physics (SMI), Vienna, Austria

49: Also at Adiyaman University, Adiyaman, Turkey

50: Also at Istanbul Aydin University, Istanbul, Turkey

51: Also at Mersin University, Mersin, Turkey

52: Also at Piri Reis University, Istanbul, Turkey

53: Also at Gaziosmanpasa University, Tokat, Turkey

54: Also at Ozyegin University, Istanbul, Turkey

55: Also at Izmir Institute of Technology, Izmir, Turkey

56: Also at Marmara University, Istanbul, Turkey

57: Also at Kafkas University, Kars, Turkey

58: Also at Istanbul Bilgi University, Istanbul, Turkey

59: Also at Hacettepe University, Ankara, Turkey

60: Also at Rutherford Appleton Laboratory, Didcot, United Kingdom

61: Also at School of Physics and Astronomy, University of Southampton, Southampton, United Kingdom

62: Also at Monash University, Faculty of Science, Clayton, Australia

63: Also at Bethel University, St. Paul, U.S.A.

64: Also at Karamanoğlu Mehmetbey University, Karaman, Turkey

65: Also at Utah Valley University, Orem, U.S.A.

66: Also at Purdue University, West Lafayette, U.S.A.

67: Also at Beykent University, Istanbul, Turkey

68: Also at Bingol University, Bingol, Turkey

69: Also at Sinop University, Sinop, Turkey

70: Also at Mimar Sinan University, Istanbul, Istanbul, Turkey

71: Also at Texas A\&M University at Qatar, Doha, Qatar

72: Also at Kyungpook National University, Daegu, Korea 